\title{
Modelling and Nonlinear Robust Control of Longitudinal Vehicle Advanced ACC Systems
}

\author{
Yang Bin ${ }^{1}$, Keqiang $\mathrm{Li}^{2}$ and Nenglian Feng ${ }^{1}$ \\ ${ }^{1}$ Beijing University of Technology \\ ${ }^{2}$ Tsinghua University \\ China
}

\section{Introduction}

Safety and energy are two key issues to affect the development of automotive industry. For the safety issue, the vehicle active collision avoidance system is developing gradually from a high-speed adaptive cruise control (ACC) to the current low-speed stop and go (SG), and the future research topic is the ACC system at full-speed, namely, the advanced ACC (AACC) system. The AACC system is an automatic driver assistance system, in which the driver's behavior and the complex traffic environment ranging are taken into account from high-speed to low-speed. By combining the function of the high-speed ACC and low-speed SG, the AACC system can regulate the relative distance and the relative velocity adaptively between two vehicles according to the driving condition and the external traffic environment. Therefore, not only can the driver stress and the energy consumption caused by the frequent manipulation and the traffic congestion both be reduced effectively at the urban traffic environment, but also the traffic flow and the vehicle safety will be improved on the highway.

Taking the actual traffic environment into account, the velocity of vehicle changes regularly in a wide range and even frequently under SG conditions. It is also subject to various external resistances, such as the road grade, mass, as well as the corresponding impact from the rolling resistance. Therefore, the behaviors of some main components within the power transmission show strong nonlinearity, for instance, the engine operating characteristics, automatic transmission switching logic and the torque converter capacity factor. In addition, the relative distance and the relative velocity of the inter-vehicles are also interfered by the frequent acceleration/deceleration of the leading vehicle. As a result, the performance of the longitudinal vehicle full-speed cruise system (LFS) represents strong nonlinearity and coupling dynamics under the impact of the external disturbance and the internal uncertainty. For such a complex dynamic system, many effective research works have been presented. J. K. Hedrick et al. proposed an upper+lower layered control algorithm concentrating on the high-speed ACC system, which was verified through a platoon cruise control system composed of multiple vehicles [1-3]. K. Yi et al. applied some linear control methods, likes linear quadratic (LQ) and proportional-integral-derivative (PID), to design the upper and lower layer controllers independently for the high-speed ACC system [4]. In ref.[5], Omae designed the model matching control (MMC) vehicle high-speed ACC system based on the $\mathrm{H}$-infinity $\left(\mathrm{H}_{\mathrm{inf}}\right)$ robust control method. To achieve a tracking control between 
the relative distance and the relative velocity of the inter-vehicles, A. Fritz proposed a nonlinear vehicle model for the high-speed ACC system with four state variables in refs.[6, 7], and designed a variable structure control (VSC) algorithm based on the feedback linearization. In ref. [8], J.E. Naranjo used the fuzzy theory to design a coordinate control algorithm between the throttle actuator and the braking system. It has been verified on an ACC and SG cruise system. Utilizing the model predictive control (MPC) method, D. Coron designed an ACC control system for a SMART Car [9]. G. N. Bifulco applied the human artificial intelligence to study an ACC control algorithm with anthropomorphic function [10]. U. Ozguner investigated the impact of inter-vehicles communications on the performance of vehicle cruise control system [11]. J. Martinez, et al. proposed a reference model-based method, which has been applied to the ACC and SG system, and achieved an expected tracking performance at full-speed condition [12]. Utilizing the idea of hierarchical design method, P. Venhovens proposed a low-speed SG cruise control system, and it has been verified on a BMW small sedan [13]. Y. Yamamura developed an SG control method based on an existing framework of the ACC control system, and applied it to the SG cruise control [14]. Focusing on the low-speed condition of the heavy-duty vehicles, Y. Bin et al. derived a nonlinear model $[15,16]$ and applied the theory of nonlinear disturbance decoupling (NDD) and LQ to the low-speed SG system $[17,18]$.

In the previous research works, the controlled object (i.e. the dynamics of the controlled vehicle) was almost simplified as a linear model without considering its own mass, gear position and the uncertainty from external environment (likes, the change of the road grade). Furthermore, the analysis of the disturbance from the leading vehicle's acceleration/ deceleration was not paid enough consideration, which has become a bottleneck in limiting the enhancement of the control performance. To summarize, based on a detailed analysis of the impact from the practical high/low speed operating condition, the uncertainty of complex traffic environment, vehicle mass, as well as the change of gear shifting to the vehicle dynamic, an innovative LFS model is proposed in this study, in which the dynamics of the controlled vehicle and the inter-vehicles are lumped together within a more accurate and reasonable mathmatical description. For the uncertainty, strong nonlinearity and the strong coupling dynamics of the proposed model, an idea of the step-by-step transformation and design is adopted, and a disturbance decoupling robust control (DDRC) method is proposed by combining the theory of NDD and VSC. On the basis of this method, it is possible to weaken the matching condition effectively within the invariance of VSC, and decouple the system from the external disturbance completely while with a simplified control structure. By this way, an improved AACC system for LFS based on the DDRC method is designed. Finally, a simulation in view of a typical vehicle moving scenario is conducted, and the results demonstrate that the proposed control system not only achieves a global optimization by means of a simplified control structure, but also exhibits an expected dynamic response, high tracking accuracy and a strong robustness regarding the external disturbance from the leading vehicle's frequent acceleration/deceleration and the internal uncertainty of the controlled vehicle.

\section{LFS model}

The LFS is composed of a leading vehicle and a controlled vehicle, and the block diagram is shown in Figure 1. The controlled vehicle is a heavy-duty truck, whose power transmission is composed of an engine, torque converter, automatic transmission and a final drive. The 
brake system is a typical one with the assistance of the compressed air. On-board millimetric wave radar is used to detect the information from the inter-vehicles (i.e., the relative distance and the relative velocity), which is installed in the front-end frame bumper of the controlled vehicle.

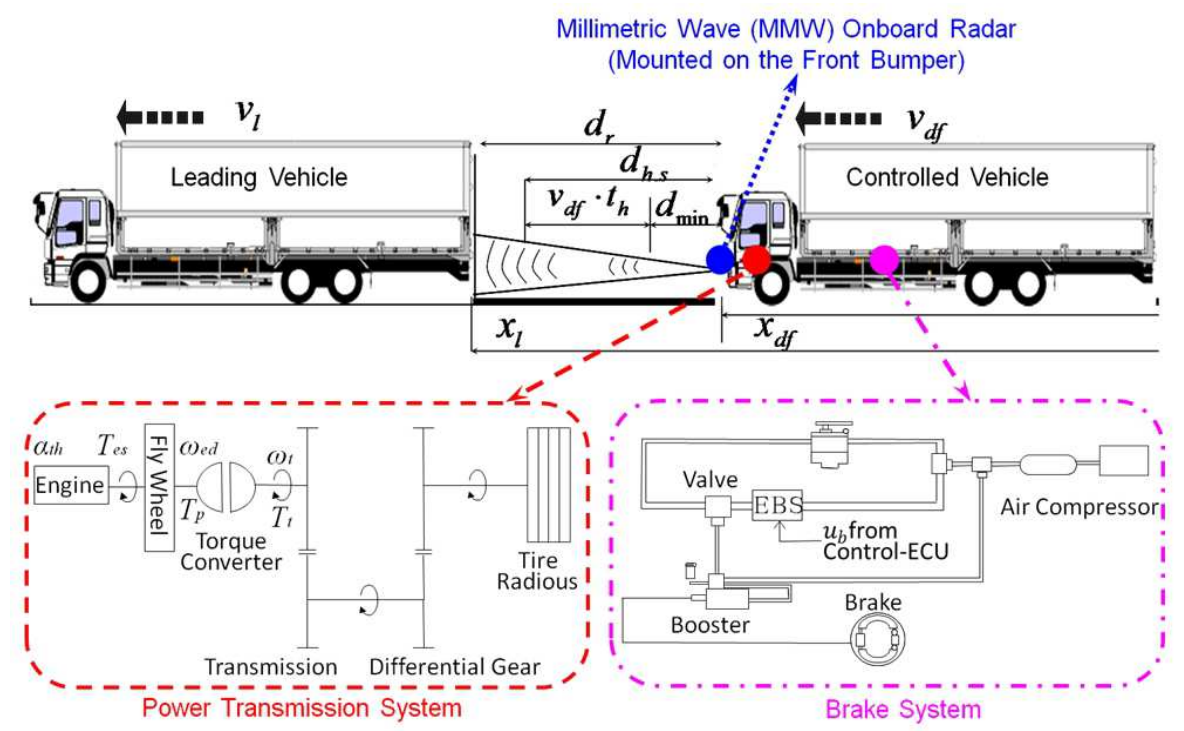

Fig. 1. Block diagram of LFS

$x_{l}, x_{d f}, v_{l}, v_{d f}$ are absolute distance $(\mathrm{m})$ and velocity $(\mathrm{m} / \mathrm{s})$ between the leading vehicle and the controlled vehicle, respectively. $d_{r}=x_{l}-x_{d f}$ is an actual relative distance between the two vehicles. Desired relative distance can be expressed as $d_{h, s}=d_{\min }+v_{d f} t_{h}$, where, $d_{\min }=5 \mathrm{~m}, t_{h}=2 \mathrm{~s}$. $v_{r}=v_{l}-v_{d f}$ is an actual relative velocity. The purpose of LFS is to achieve the tracking of the inter-vehicles relative distance/relative velocity along a desired value. Therefore, a dynamics model of LFS at low-speed condition has been derived in ref. [15], which consists of two parts. The first part is the longitudinal dynamics model of the controlled vehicle, in which the nonlinearity of some main components, such as the engine, torque converter, etc, is taken into account. However, this model is only available at the following strict assumptions:

- $\quad$ the vehicle moves on a flat straight road at a low speed $(<7 \mathrm{~m} / \mathrm{s})$

- $\quad$ assume the mass of vehicle body is constant

- the automatic transmission gear box is locked at the first gear position

- $\quad$ neglect the slip and the elasticity of the power train

The second part is the longitudinal dynamics model of the inter-vehicles, in which the disturbance from frequent accelartion/deccelartion of the leading vehicle is considered.

In general, since the mass, road grade and the gear position of the automatic transmission change regularly under the practical driving cycle and the traffic environment, the longitudinal dynamics model of the controlled vehicle in ref. [15] can only be used in some way to deal with an ideal traffic environment (i.e., the low-speed urban condition). In view of the uncertainties above, in this section, a more accurate longitudinal dynamics model of 
the controlled vehicle is derived for the purpose for high-speed and low-speed conditions (that is, the full-speed condition). After that, it will be integrated with a longitudinal dynamics model of the inter-vehicles, and an LFS dynamics model for practical applications can be obtained in consideration of the internal uncertainty and the external disturbance. It is a developed model with enhanced accuracy, rather than a simple extension in contrast with ref. [15].

\subsection{Longitudinal dynamics model of the controlled vehicle}

Based on the vehicle multi-body dynamics theory [19], modeling principles, and the above assumptions, two nominal models of the longitudinal vehicle dynamics are derived firstly according to the driving/braking condition:

The driving condition:

$$
\dot{\boldsymbol{X}}=\boldsymbol{F}_{a v}(\boldsymbol{X})+\boldsymbol{G}_{a v}(\boldsymbol{X}) \alpha_{t h} \Rightarrow\left[\begin{array}{c}
\dot{x}_{1} \\
\dot{x}_{2}
\end{array}\right]=\left[\begin{array}{c}
f_{a v 1}(\boldsymbol{X}) \\
f_{a v 2}(\boldsymbol{X})
\end{array}\right]+\left[\begin{array}{c}
g_{a v 1}(\boldsymbol{X}) \\
g_{a v 2}(\boldsymbol{X})
\end{array}\right] \alpha_{t h}
$$

where two state variables are $x_{1}=\omega_{t}$ (turbine speed $(\mathrm{r} / \mathrm{min})$ ) and $x_{2}=\omega_{\text {ed }}$ (engine speed $(\mathrm{r} / \mathrm{min}))$; a control variable is $a_{t h}$ (percentage of the throttle angle (\%)); definitions of nonlinear items $f_{a v 1}(\boldsymbol{X}), f_{a v 2}(\boldsymbol{X}), g_{a v 1}(\boldsymbol{X})$ and $g_{a v 2}(\boldsymbol{X})$ are presented in Appendix (1).

The braking condition:

$$
\dot{\boldsymbol{X}}=\boldsymbol{F}_{d v}(\boldsymbol{X})+\boldsymbol{G}_{d v}(\boldsymbol{X}) u_{b} \Rightarrow\left[\begin{array}{c}
\dot{x}_{1} \\
\dot{x}_{2} \\
\dot{x}_{3}
\end{array}\right]=\left[\begin{array}{c}
f_{d v 1}(\boldsymbol{X}) \\
f_{d v 2}(\boldsymbol{X}) \\
f_{d v 3}(\boldsymbol{X})
\end{array}\right]+\left[\begin{array}{l}
g_{d v 1}(\boldsymbol{X}) \\
g_{d v 2}(\boldsymbol{X}) \\
g_{d v 3}(\boldsymbol{X})
\end{array}\right] u_{b}
$$

where $x_{3}=a_{b}$ is a braking deceleration $\left(\mathrm{m} / \mathrm{s}^{2}\right) ; u_{b}$ is a control variable of the desired input voltage of EBS $(V)$; definitions of nonlinear items $f_{d v 1}(\boldsymbol{X}) \sim f_{d v 3}(\boldsymbol{X})$ and $g_{d v 1}(\boldsymbol{X}) \sim g_{d v 3}(\boldsymbol{X})$ are presented in Appendix (2).

As mentioned earlier, models (1) and (2) are available based upon some strict assumptions. In view of the actual driving condition and complex traffic environment, some uncertainties which this heavy-duty vehicle may possibly encounter can be presented as follows:

1. variation of the mass $10,000 \mathrm{~kg} \leq M \leq 25,000 \mathrm{~kg}$

2. variation of the road grade $-3^{\circ} \leq \varphi_{s} \leq 3^{\circ}$

3. gear position shifting of the automatic transmission $i_{81}=3.49, i_{82}=1.86, i_{83}=1.41, i_{g 4}=1$, $i_{g 5}=0.7, i_{g 6}=0.65$.

4. mathematical modeling error from the engine, torque converter and the heat fade efficiency of the braking system.

Considering the uncertainties above, two longitudinal dynamics models of the controlled vehicle differ from Eqs. (1) and (2) are therefore expressed as

Driving condition:

$$
\dot{\boldsymbol{X}}=\left[\boldsymbol{F}_{a v}(\boldsymbol{X})+\Delta \boldsymbol{F}_{a v}(\boldsymbol{X})\right]+\left[\boldsymbol{G}_{a v}(\boldsymbol{X})+\Delta \boldsymbol{G}_{a v}(\boldsymbol{X})\right] \alpha_{t h}
$$

Braking condition:

$$
\dot{\boldsymbol{X}}=\left[\boldsymbol{F}_{d v}(\boldsymbol{X})+\Delta \boldsymbol{F}_{d v}(\boldsymbol{X})\right]+\left[\boldsymbol{G}_{d v}(\boldsymbol{X})+\Delta \boldsymbol{G}_{d v}(\boldsymbol{X})\right] u_{b}
$$


where $\Delta \boldsymbol{F}_{a v}(\boldsymbol{X}), \Delta \boldsymbol{G}_{a v}(\boldsymbol{X}), \Delta \boldsymbol{F}_{d v}(\boldsymbol{X}), \Delta \boldsymbol{G}_{d v}(\boldsymbol{X})$ are system uncertain matrixes relative to the nominal model. They are influenced by various factors, and are described as

$$
\Delta \boldsymbol{F}_{a v}(\boldsymbol{X})=\left[\begin{array}{c}
\Delta f_{a v 1} \\
\Delta f_{a v 2}
\end{array}\right], \Delta \boldsymbol{G}_{a v}(\boldsymbol{X})=\left[\begin{array}{c}
\Delta g_{a v 1} \\
\Delta g_{a v 2}
\end{array}\right], \Delta \boldsymbol{F}_{d v}(\boldsymbol{X})=\left[\begin{array}{c}
\Delta f_{d v 1} \\
\Delta f_{d v 2} \\
\Delta f_{d v 3}
\end{array}\right], \quad \Delta \boldsymbol{G}_{d v}(\boldsymbol{X})=\left[\begin{array}{c}
\Delta g_{d v 1} \\
\Delta g_{d v 2} \\
\Delta g_{d v 3}
\end{array}\right] .
$$

In terms of multiple factors of the uncertain matrixes, it is difficult to estimate the upper and lower boundaries of Eqs. (3) and (4) precisely by using the mathematical analytic method. Therefore, a simulation model of the heavy-duty vehicle is created at first by using the MATLAB/Simulink software, which will be used to estimate the upper and lower boundaries of the uncertain matrixes. To determine the upper and lower boundaries, an analysis on extreme driving/breaking conditions of models (3) and (4) is.

At first, the analysis of Eq. (3) indicates that with the increase of the mass $M$, road grade $\varphi_{\mathrm{s}}$ and the gear position, the item of $f_{a v 1}(X)$ converges reversely to its minimum value relative to the nominal condition (at a given $\omega_{t}, \omega_{e d}$ ). Similarly, the extreme operating condition for the maximum value of $f_{a v 1}(X)$ can be obtained. The analysis above can be applied equally to other items of Eq. (3), and can be summarized as the following two extreme conditions:

(a1) If the vehicle mass is $M=10,000 \mathrm{~kg}$, the road grade is $\varphi_{s}=-3^{\circ}$ and the automatic transmission is locked at the first gear position, then the upper boundary of $\Delta f_{a v 1}$ can be estimated.

(a2) If the vehicle mass is $M=25,000 \mathrm{~kg}$, the road grade is $\varphi_{s}=-3^{\circ}$ and the automatic transmission is shifted to the third gear position (supposing that the gear position can not be shifted up to the sixth gear position, since it should be subject to a known gear shift logic under a given actual traffic condition), then the lower boundary of $\Delta f_{a v 1}$ can be estimate.

On the analysis of Eq. (4), two extreme conditions corresponding to the upper and lower boundaries can also be obtained:

(b1) If the vehicle mass is $M=10,000 \mathrm{~kg}$, the road grade is $\varphi_{s}=-3^{\circ}$, the braking deceleration is $a_{b}=0 \mathrm{~m} / \mathrm{s}^{2}$ and the gear position is locked at the first gear position, then the upper boundary of $\Delta f_{d v 1}$ can be estimated.

(b2) If the vehicle mass is $M=25,000 \mathrm{~kg}$, the road grade is $\varphi_{\mathrm{s}}=3^{\circ}$, the braking deceleration is $a_{b}=-2 \mathrm{~m} / \mathrm{s}^{2}$ (assuming it as the maximum braking deceleration commonly used) and the gear position is locked at the third gear position, then the lower boundary of $\Delta f_{d v 1}$ can be estimated.

By the foregoing analysis, the extreme and nominal operating conditions will be simulated respectively by using the simulation model of the heavy-duty vehicles. In order to activate entire gear positions of the automatic transmission, the vehicle is accelerated from $0 \mathrm{~m} / \mathrm{s}$ to the maximum velocity by inputting a engine throttle percentage of $100 \%$. After that, the throttle angle percentage is closed to $0 \%$, and the velocity is slowed down gradually in the following two patterns:

1. according to the requirement of (b1) condition, the vehicle is slowed down until stop by the use of the engine invert torque and the road resistance.

2. according to the requirement of (b2) condition, the vehicle is slowed down until stop through an adjoining of a deceleration $a_{b}=-2 \mathrm{~m} / \mathrm{s}^{2}$ generated by the EBS, as well as the sum of the engine invert torque and the road resistance. 
According to the above extreme conditions (a1), (a2), (b1), (b2), the variation range of each uncertainty can be obtained by simulation, as shown in Figures 2 and 3 . For removing the influence from the gear position, the $x$-coordinates in Figures 2 and 3 have been transferred into a universal scale of the engine speed.

For instance (see solid line in Figure 2), during the increase of the engine speed in condition (a1), the upper boundary of the item $\Delta f_{a v 1}$ increases gradually, while the items $\Delta f_{a v 2}, \Delta g_{a v 2}$ change trivially. As to the increase of the engine speed in condition (a2) (see dashed line in Figure 2), the lower boundary of the item $\Delta f_{a v 1}$ increases rapidly at the beginning, and then drops slowly. The minimum value appears approximately at the slowest speed of the engine (i.e., the idle condition). The items $\Delta f_{a v 2}, \Delta g_{a v 2}$ decrease during the engine speed increases.

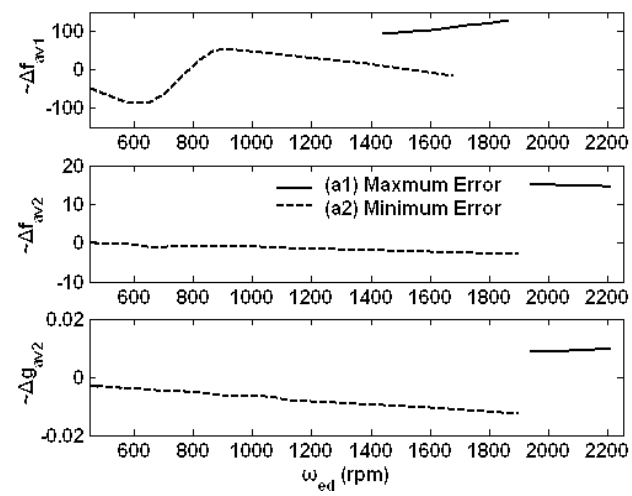

Fig. 2. Changes of uncertain items under driving condition

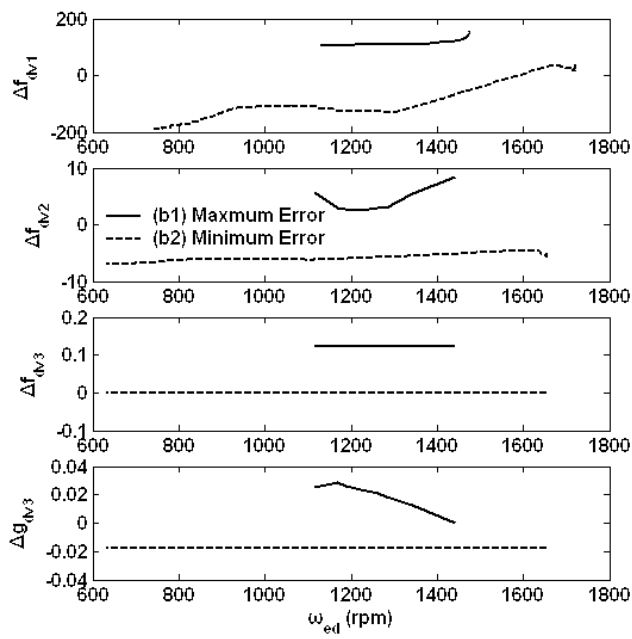

Fig. 3. Changes of uncertain items under braking condition

From the above simulation results, it is easy to calculate the upper and lower boundaries of the uncertain matrixes in Eqs. (3) and (4): 
Driving condition:

$$
-86 \leq \Delta f_{a v 1} \leq 127, \quad-2.75 \leq \Delta f_{a v 2} \leq 15, \quad \Delta g_{a v 1}=0, \quad-0.0127 \leq \Delta g_{a v 2} \leq 0.001 .
$$

Braking condition:

$$
\begin{aligned}
& -188 \leq \Delta f_{d v 1} \leq 155,-7 \leq \Delta f_{d v 2} \leq 8.45,0 \leq \Delta f_{d v 3} \leq 0.124, \Delta g_{d v 1}=\Delta g_{d v 2}=0, \\
& -0.0174 \leq \Delta g_{d v 3} \leq 0.029
\end{aligned}
$$

where a unit of $\Delta f_{a^{*}}, \Delta f_{d^{*}}$ is $\mathrm{r} / \mathrm{min}^{2}$, two units of $\Delta g_{a^{*}}, \Delta g_{d^{*}}$ are $\mathrm{r} /\left(\mathrm{min}^{2} \cdot \%\right)$ and $\mathrm{m} /\left(\mathrm{s}^{3} \cdot \mathrm{V}\right)$, respectively.

To verify the proposed models, some profiles are prepared in Figure 4 according to the aforementioned extreme conditions. They include the throttle angle percentage, EBS desired braking voltage and the road grade containing two values of $\pm 3^{\circ}$. Figures 5 and 6 are the
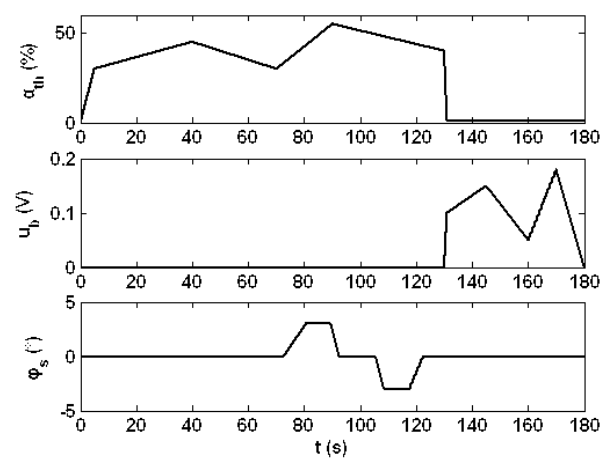

Fig. 4. Profiles of throttle angle percentage, EBS desired braking voltage and road grade
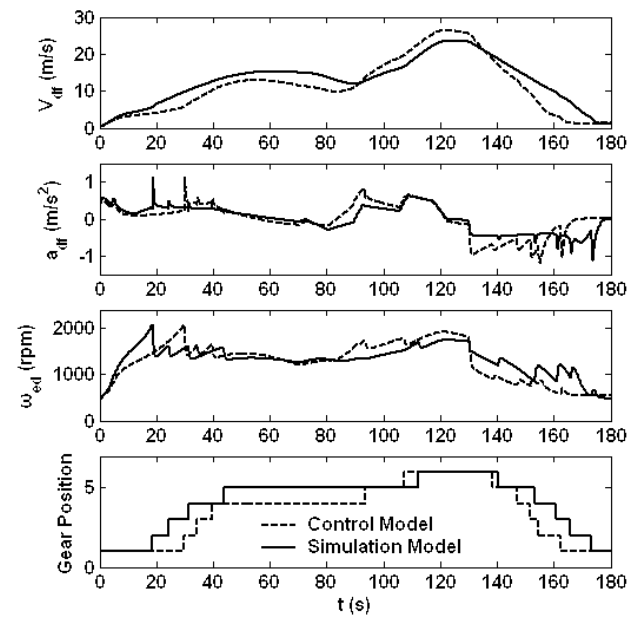

Fig. 5. Comparison results between control and simulation models $(10,000 \mathrm{~kg})$ 

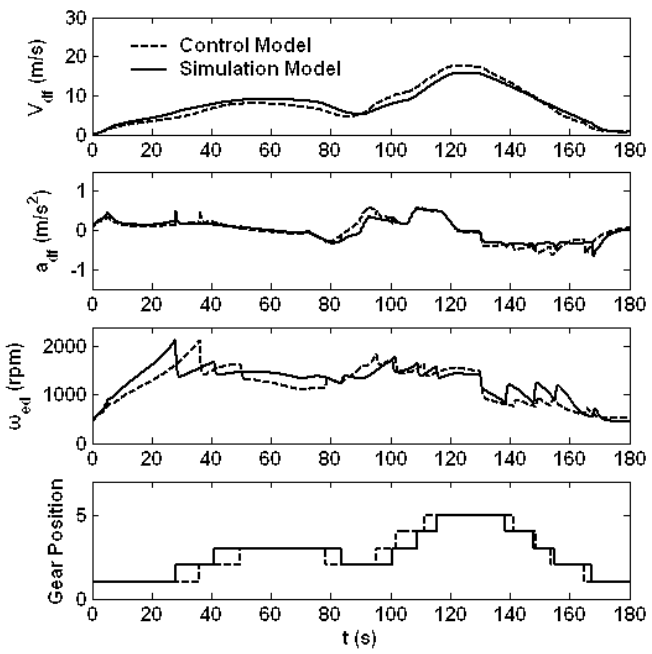

Fig. 6. Comparison results between control and simulation models $(25,000 \mathrm{~kg})$

comparison results corresponding to $10,000 \mathrm{~kg}$ and $25,000 \mathrm{~kg}$, respectively. The dashed lines and the solid lines are the results of the control models (3) and (4) and the simulation models, respectively. It can be seen from the comparison results that the control models (3) and (4) are able to approximate the simulation models very closely, even in the case of a wide variation ranges of the velocity $(0 \mathrm{~m} / \mathrm{s} \sim 28 \mathrm{~m} / \mathrm{s})$, mass $(10,000 \mathrm{~kg} \sim 25,000 \mathrm{~kg})$ and the gear positions of the automatic transmission (1 6 gears). Because the models (3) and (4) only present the longitudinal dynamics of the controlled vehicle, the inter-vehicles dynamics has to be considered furthermore such that a completed dynamics model of the LFS at fullspeed can be obtained.

\subsection{Longitudinal dynamic model of the inter-vehicles}

For the purposes of vehicular ACC or SG cruise control system design, many well-known achievements on the operation policy for the inter-vehicles relative distance and velocity have been intense studied [20,21]. Focusing on the AACC system, the operation policy for the inter-vehicles relative distance and relative velocity should be determined so as to

- maintain desirable spacing between the vehicles

- $\quad$ ensure string stability of the convoy

Inspired by previous research [1], [2], [7] on the design of upper level controller, the operation policy of inter-vehicles relative distance and relative velocity can be defined as

$$
\begin{aligned}
& \varepsilon_{d}=d_{h, s}-d_{r}=\left(d_{\min }+v_{d f} t_{h}\right)-\left(x_{l}-x_{d f}\right) \\
& \varepsilon_{v}=a_{d f} t_{h}-v_{r}=a_{d f} t_{h}-\left(v_{l}-v_{d f}\right)
\end{aligned} .
$$

where $a_{d f}$ is a controlled vehicle acceleration $\left(\mathrm{m} / \mathrm{s}^{2}\right) ; \varepsilon_{d}$ is a tracking error of the longitudinal relative distance $(\mathrm{m}) ; \varepsilon_{v}$ is a tracking error of the longitudinal relative velocity $(\mathrm{m} / \mathrm{s})$.

As the illustration of the vehicle longitudinal AACC system (see Figure 1), it should be noted that an item $a_{d f} t_{h}$ is introduced to define the inter-vehicles relative velocity $\varepsilon_{v}$ so as to 
fit the dynamical process from one stable state to another one. In contrast to Eq. (5), conventional operation policy of inter-vehicles relative velocity is often defined as $\varepsilon_{v}=v_{l}-v_{d f}$, which only focuses on the static situation of invariable velocity following. However, on account of the dynamic situation of acceleration/deceleration, the previously investigation $[15,16]$ has demonstrated that it is dangerous and uncomfortable for the AACC system to track a vehicle in front still adopted conventional operation policy. Therefore, an item of $a_{d f} t_{h}$ is proposed to capture accurately the human driver's longitudinal behavior aiming at this situation. Generally, Eq. (5) can be regarded as the dynamical operation policy.

The accuracy of Eq. (5) is validated by the following experimental tests, which is carried out under complicated down-town traffic conditions in terms of five skillful adult drivers (including four males and one female). Two cases including an acceleration tracking and a deceleration approaching are considered. In the case of acceleration tracking, the driver is closing up a leading vehicle without initial error of relative distance and relative velocity. Then, the driver adjusts his/her velocity to the one of the vehicle in front. The headway distance aimed at by the driver during the tracking is essentially depending on the driver's desire of safety. In the case of deceleration approaching, the driver is closing down a leading vehicle with constant velocity. The driver brakes to reestablish the minimal headway distance, and then follow the leading vehicle with the same velocity. The experimental data presented in Figure 7 are the mean square value of five drivers' results. The comparison results confirm that Eq. (5) shows a sufficient agreement with practical driver manipulation, which can be adopted in the design of vehicle longitudinal AACC system.

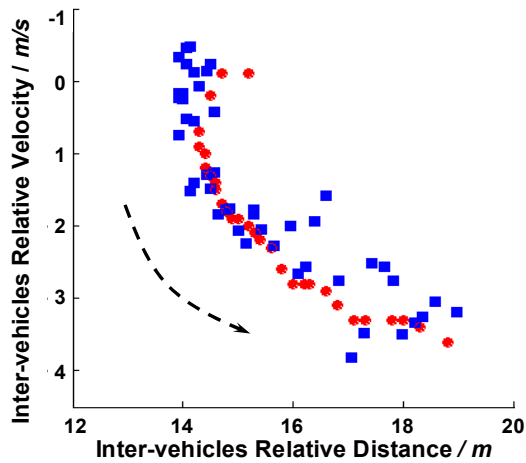

-Operation Policy •Experimental Data

(a) Acceleration tracking condition

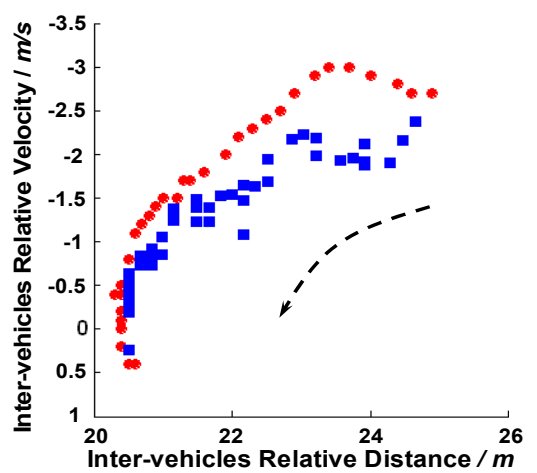

(b) Deceleration approaching condition

Fig. 7. Comparison results between experimental data and operation policy

By virtue of the operation policy (5), the mathematical model of inter-vehicles longitudinal dynamics is created

$$
\begin{aligned}
& \dot{\varepsilon}_{d}=\varepsilon_{v}=a_{d f} t_{h}-\left(v_{l}-v_{d f}\right) \\
& \dot{\varepsilon}_{v}=\dot{a}_{d f} t_{h}-\left(\dot{v}_{l}-\dot{v}_{d f}\right)
\end{aligned} .
$$

where $\dot{v}_{l}$ is a leading vehicle acceleration $\left(\mathrm{m} / \mathrm{s}^{2}\right)$, which is generally limited within an extreme acceleration/deceleration condition, i.e., $-2 \mathrm{~m} / \mathrm{s}^{2} \leq \dot{v}_{l} \leq 2 \mathrm{~m} / \mathrm{s}^{2}$. 
Although the inter-vehicles dynamics is considered in Eq. (6), the dynamics of the controlled vehicle that has great impact on the performance of entire system has been ignored instead. Actually, two aforementioned models are interrelated and coupled mutually in the LFS. To overcome the disadvantages of the existing independent modeling method, a more accurate model will be proposed in the following to describe the dynamics of the LFS reasonably. In this model, the longitudinal vehicle dynamics models (3) and (4) with uncertainty and the longitudinal inter-vehicles dynamic model (6) are both taken into account. As a result, a control system can be designed on this platform, and an optimal tracking performance with better robustness can also be achieved.

\subsection{LFS dynamics model}

Firstly, take the time derivative of the state variable $\dot{\omega}_{t}$ in Eq. (3), and obtain $\ddot{\omega}_{t}$. After that, $\dot{\omega}_{t}, \ddot{\omega}_{t}$ are substituted into Eq. (6) by virtue of the relationship $a_{d f}=\alpha_{n} \dot{\omega}_{t}=\frac{2 \pi r_{t}}{60 i_{g} i_{0}} \dot{\omega}_{t}$. Finally, an LFS dynamics model for the driving condition is derived according to Eqs. (3) and (6). It is a combination of the dynamics between the controlled vehicle and the inter-vehicles, as well as the uncertainty from actual driving conditions.

$$
\begin{aligned}
& \dot{\boldsymbol{X}}=\left[\boldsymbol{F}_{a}(\boldsymbol{X})+\Delta \boldsymbol{F}_{a}(\boldsymbol{X})\right]+\left[\boldsymbol{G}_{a}(\boldsymbol{X})+\Delta \boldsymbol{G}_{a}(\boldsymbol{X})\right] \alpha_{t h}+\boldsymbol{P}_{a}(\boldsymbol{X}) w \\
& \Leftrightarrow\left\{\begin{array}{l}
\dot{\varepsilon}_{d}=f_{a 1} \\
\dot{\varepsilon}_{v}=\left(f_{a 2}+\Delta f_{a 2}\right)+\left(g_{a 1}+\Delta g_{a 1}\right) \alpha_{t h}+p_{a 1} \dot{v}_{l} \\
\dot{\omega}_{t}=f_{a 3}+\Delta f_{a 3} \\
\dot{\omega}_{e d}=\left(f_{a 4}+\Delta f_{a 4}\right)+\left(g_{a 2}+\Delta g_{a 2}\right) \alpha_{t h}
\end{array}\right.
\end{aligned}
$$

where $X=\left[\begin{array}{llll}\varepsilon_{d} & \varepsilon_{v} & \omega_{t} & \omega_{e d}\end{array}\right]^{T}$ is a vector of the state variables, $w=\dot{v}_{l}$ is a disturbance variable, and $\alpha_{t h}$ is a control variable. The definition of each item in Eq. (7) can be referred to Appendix (2).

Similarly, an LFS dynamics model for the braking condition is achieved:

$$
\begin{aligned}
& \dot{\boldsymbol{X}}=\left[\boldsymbol{F}_{d}(\boldsymbol{X})+\Delta \boldsymbol{F}_{d}(\boldsymbol{X})\right]+\left[\boldsymbol{G}_{d}(\boldsymbol{X})+\Delta \boldsymbol{G}_{d}(\boldsymbol{X})\right] u_{b}+\boldsymbol{P}_{d}(\boldsymbol{X}) w \\
& \Leftrightarrow\left\{\begin{array}{l}
\dot{\varepsilon}_{d}=f_{d 1} \\
\dot{\varepsilon}_{v}=\left(f_{d 2}+\Delta f_{d 2}\right)+\left(g_{d 1}+\Delta g_{d 1}\right) u_{b}+p_{d 1} \dot{\boldsymbol{v}}_{l} \\
\dot{\omega}_{t}=f_{d 3}+\Delta f_{d 3} \\
\dot{\omega}_{e d}=f_{d 4}+\Delta f_{d 4} \\
\dot{a}_{b}=\left(f_{d 5}+\Delta f_{d 5}\right)+\left(g_{d 2}+\Delta g_{d 2}\right) u_{b}
\end{array}\right.
\end{aligned}
$$

where $X=\left[\begin{array}{lllll}\varepsilon_{d} & \varepsilon_{v} & \omega_{t} & \omega_{e d} & a_{b}\end{array}\right]^{T}$ is a vector of the state variables, $u_{b}$ is a control variable. The definition of each item in Eq. (8) can be referred to Appendix (4).

According to the analysis of the extreme driving/braking conditions in 2.1, an approximate ranges of the upper and lower boundaries regarding uncertain items in Eqs. (7) and (8) can be calculated through simulation. 
Driving condition:

Braking condition:

$$
-104 \leq \Delta f_{a 2} \leq 203,-0.031 \leq \Delta g_{a 1} \leq 0.0027
$$

$$
-192 \leq \Delta f_{d 2} \leq 174,-0.0153 \leq \Delta g_{d 1} \leq 0.022
$$

where an unit of $\Delta f_{*}$ is $\mathrm{m} / \mathrm{s}^{2}$, units of $\Delta g_{a 1}, \Delta g_{d 1}$ are $\mathrm{m} /\left(\mathrm{s}^{2} \% \%\right)$ and $\mathrm{m} /\left(\mathrm{s}^{2} \cdot V\right)$, respectively. The analysis of the dynamics models (7) and (8) indicates that the LFS is an uncertain affine nonlinear system, in which the strong nonlinearities and the coupling properties caused by the disturbance and the uncertainty are represented. These complex behaviors result in more difficulties while implementing the control of the LFS, since the state variables $\varepsilon_{d}, \varepsilon_{v}$ are influenced significantly by the nonlinearity, uncertainty, as well as the disturbance from the leading vehicle's acceleration/deceleration. However, because the longitudinal dynamics of the controlled vehicle and the inter-vehicles can be described and integrated into a universal frame of the state space equation accurately, this would be helpful for the purpose of achieving a global optimal and a robust control for the LFS.

The LFS AACC system intends to implement the accurate tracking control of the intervehicles relative distance/relative velocity under both high-speed and crowded traffic environments. Thus, the system should be provided with strong robustness in view of the complex external disturbance and the internal uncertainty, as well as the capability to eliminate the impact from the system's strong nonlinearity at low-speed. Focusing on the LFS, refs. [22-27] presented an NDD method to eliminate the disturbance effectively, which was, however, limited to some certain affine nonlinear systems. Utilizing the invariance of the sliding mode in VSC, the control algorithm proposed in refs. [28, 29] can implement the completely decoupling of all state variables from the disturbance and the uncertainty. But, it is not a global decoupling algorithm, and should also be submitted to some strict matching conditions. Refs. [30-34] studied the input-output linearization on an uncertain affine nonlinear system, but did not discuss the disturbance decoupling problem. On a nonlinear system with perturbation, ref. [35] gave the necessary and sufficient condition for the completely disturbance decoupling problem, but did not present the design of the feedback controller. To avoid the disadvantages of those control algorithms mentioned above, a DDRC method combining the theory of NDD and VSC is proposed in regard to the complex dynamics of the LFS.

\section{DDRC method}

The basic theory of DDRC method is inspired by the idea of the step-by-step transformation and design. First, on account of a certain affine nonlinear system with disturbance, the NDD theory based on the differential geometry is used to implement the disturbance decoupling and the input-output linearization. Hence, a linearized subsystem with partial state variables is given, in which the invariance matching conditions of the sliding mode can be discussed easily via VSC theory, and then a VSC controller can be deduced. Finally, two methods will be integrated together such that a completely decoupling of the system from the external disturbance, and a weakened invariance matching condition with a simplified control system structure are obtained. 


\subsection{NDD theory on certain affine nonlinear system}

At first, consider a certain dynamics model of the LFS, where uncertain items of $\Delta \boldsymbol{F}_{a}(\boldsymbol{X})$, $\Delta \boldsymbol{G}_{a}(\boldsymbol{X}), \Delta \boldsymbol{F}_{d}(\boldsymbol{X})$ and $\Delta \boldsymbol{G}_{d}(\boldsymbol{X})$ are considered as zero. Hence, a certain affine nonlinear system can be simplified as

$$
\left\{\begin{array}{l}
\dot{X}=\boldsymbol{F}(\boldsymbol{X})+\boldsymbol{G}(\boldsymbol{X}) u+\boldsymbol{P}(\boldsymbol{X}) w \\
y=h(X)
\end{array}\right.
$$

where $\boldsymbol{X} \in R^{n}$ and $u, w, y \in R$ are system state variable, control variable, disturbance variable and output variable, respectively, $F, G, P, h$ are differentiable functions of $X$ with corresponding dimensions.

The basic theory of NDD is trying to seek a state feedback, and construct a closed-loop system as follows

$$
\left\{\begin{array}{l}
\dot{\boldsymbol{X}}=\boldsymbol{F}(\boldsymbol{X})+\boldsymbol{G}(\boldsymbol{X}) \alpha(\boldsymbol{X})+\boldsymbol{G}(\boldsymbol{X}) \beta(\boldsymbol{X}) v+\boldsymbol{P}(\boldsymbol{X}) w=\tilde{\boldsymbol{F}}(\boldsymbol{X})+\tilde{\boldsymbol{G}}(\boldsymbol{X}) v+\boldsymbol{P}(\boldsymbol{X}) w \\
y=h(\boldsymbol{X})
\end{array}\right.
$$

If there is an invariant distribution $\Delta(X)$ that exists over $\tilde{F}(X), \tilde{G}(X)$, and satisfies

$$
\operatorname{span}\{\boldsymbol{P}\} \subset \Delta(X) \subset \Omega^{\perp}(X)
$$

where

$$
\Omega(\boldsymbol{X})=\left[\begin{array}{llll}
d h(\boldsymbol{X}) & d L_{\boldsymbol{F}} h(\boldsymbol{X}) & \cdots & d L_{\boldsymbol{F}}^{r-1} h(\boldsymbol{X})
\end{array}\right]^{T} .
$$

Then, the output $y$ can be decoupled from the disturbance $w$, and we have a $r$-dimension coordinate transformation

$$
\mathbf{Z}=\boldsymbol{\psi}(\boldsymbol{X})=\left[z_{1}, \cdots, z_{r}\right]^{T}=\left[h(\boldsymbol{X}), \cdots, L_{\boldsymbol{F}}^{r-1} h(\boldsymbol{X})\right]^{T}
$$

as well as an $n$-r-dimension coordinate transformation

$$
\boldsymbol{\mu}=\boldsymbol{\phi}(\boldsymbol{X})=\left[\mu_{1}(\boldsymbol{X}), \cdots, \mu_{n-r}(\boldsymbol{X})\right]^{T}
$$

where $\mu$ satisfies

$$
d \mu_{i}(X) G(X)=0, \quad \forall X \in U, \quad i=1, \cdots, n-r
$$

In this way, the original closed-loop system (9) can be modified as a following form over the new coordinate

$$
\begin{aligned}
& \dot{z}_{i}=z_{i+1} \quad 1 \leq i \leq r-1 \\
& \dot{z}_{r}=v \\
& \dot{\boldsymbol{\mu}}=\boldsymbol{Q}(\mathbf{Z}, \boldsymbol{\mu})+\boldsymbol{K}(\mathbf{Z}, \boldsymbol{\mu}) w
\end{aligned}
$$


Obviously, Eq. (15) is a linearized decoupling subsystem, while Eq. (16) is a nonlinear internal dynamic subsystem subject to the disturbance. The invariant distribution $\Delta(X)$ is defined as $[\tilde{\boldsymbol{F}}, \Delta](\boldsymbol{X}) \subset \Delta(\boldsymbol{X}), L$ is a Lie derivative, defined as $L_{\boldsymbol{F}} G=\left(\frac{\partial \boldsymbol{G}}{\partial \boldsymbol{X}}\right) \boldsymbol{F}, r$ is a relative degree, defined as $L_{G} L_{F}^{r-1} h(X) \neq 0$ [36], $*^{\perp}$ is an orthogonal of" * "[37]. Eq. (10) is a necessary and sufficient condition of the disturbance decoupling problem, which can be expressed in the equivalent form

$$
\Omega(X) P(X)=0
$$

State feedback is

$$
u=\alpha(\boldsymbol{X})+\beta(\boldsymbol{X}) v=\frac{-L_{F}^{r} h(\boldsymbol{X})+v}{L_{G} L_{\boldsymbol{F}}^{r-1} h(\boldsymbol{X})}
$$

If the disturbance $w$ is measurable, the following state feedback can be considered

$$
u=\alpha(\boldsymbol{X})+\beta(\boldsymbol{X}) v+\gamma(\boldsymbol{X}) w=\frac{-L_{\boldsymbol{F}}^{r} h(\boldsymbol{X})+v-L_{P} L_{F}^{r-1} h(\boldsymbol{X}) w}{L_{G} L_{F}^{r-1} h(X)}
$$

In this way, a weakened necessary and sufficient condition of the disturbance decoupling problem is achieved as

$$
\Omega(X)[G(X) \gamma+P(X)]=\mathbf{0}
$$

As a result, some existing linear control methods (likes, LQ, pole placement) can be used to implement the pole placement over the linearized decoupling subsystem. In the following, the NDD theory is used to discuss the VSC problem of the affine nonlinear systems under the impact of the uncertainty.

\subsection{VSC of uncertain affine nonlinear systems based on NDD}

Considering Eqs. (7) and (8) with uncertainty, they can be simplified as a more general forms for the analysis, i.e.,

$$
\left\{\begin{array}{l}
\dot{X}=[\boldsymbol{F}(\boldsymbol{X})+\Delta \boldsymbol{F}(\boldsymbol{X})]+[\boldsymbol{G}(\boldsymbol{X})+\Delta \boldsymbol{G}(\boldsymbol{X})] u+[\boldsymbol{P}(\boldsymbol{X})+\Delta \boldsymbol{P}(\boldsymbol{X})] w \\
y=h(\boldsymbol{X})
\end{array}\right.
$$

where $\boldsymbol{F}, \boldsymbol{G}, \boldsymbol{P}, h$ indicate the certain part of the system, and they are defined as Eq. (8), $\Delta \boldsymbol{F}$, $\Delta G, \Delta \boldsymbol{P}$ indicate the uncertain part correspondingly.

At first, take first derivative of the output variable $y=h(\boldsymbol{X})$ :

$$
\begin{aligned}
& \dot{z}_{1}=\frac{d y}{d t} \\
& =\frac{\partial h(\boldsymbol{X})}{\partial \boldsymbol{X}}[\boldsymbol{F}(\boldsymbol{X})+\boldsymbol{G}(\boldsymbol{X}) u+\boldsymbol{P}(\boldsymbol{X}) w]+\frac{\partial h(\boldsymbol{X})}{\partial \boldsymbol{X}}[\Delta \boldsymbol{F}(\boldsymbol{X})+\Delta \boldsymbol{G}(\boldsymbol{X}) u+\Delta \boldsymbol{P}(\boldsymbol{X}) w] \\
& =\left[L_{\boldsymbol{F}} h(\boldsymbol{X})+L_{G} h(\boldsymbol{X}) u+L_{\boldsymbol{P}} h(\boldsymbol{X}) w\right]+\left[L_{\Delta \boldsymbol{F}} h(\boldsymbol{X})+L_{\Delta G} h(\boldsymbol{X}) u+L_{\Delta \boldsymbol{P}} h(\boldsymbol{X}) w\right]
\end{aligned}
$$


Obviously, if

$$
L_{\Delta F} h(X)=L_{\Delta G} h(X)=L_{\Delta P} h(X)=0
$$

then according to the definition of the relative degree and Eq. (17), Eq. (22) becomes

$$
\dot{z}_{1}=L_{F} h(X)=z_{2}
$$

Differentiate Eq. (24) again yields

$$
\begin{aligned}
& \dot{z}_{2}=\frac{d L_{F} h(\boldsymbol{X})}{d t} \\
& =\frac{\partial L_{\boldsymbol{F}} h(\boldsymbol{X})}{\partial \boldsymbol{X}}[\boldsymbol{F}(\boldsymbol{X})+\boldsymbol{G}(\boldsymbol{X}) u+\boldsymbol{P}(\boldsymbol{X}) w]+\frac{\partial L_{F} h(\boldsymbol{X})}{\partial \boldsymbol{X}}[\Delta \boldsymbol{F}(\boldsymbol{X})+\Delta \boldsymbol{G}(\boldsymbol{X}) u+\Delta \boldsymbol{P}(\boldsymbol{X}) w] \\
& =\left[L_{F}^{2} h(\boldsymbol{X})+L_{G} L_{\boldsymbol{F}} h(\boldsymbol{X}) u+L_{\boldsymbol{P}} L_{\boldsymbol{F}} h(\boldsymbol{X}) w\right]+\left[L_{\Delta F} L_{\boldsymbol{F}} h(\boldsymbol{X})+L_{\Delta G} L_{F} h(\boldsymbol{X}) u+L_{\Delta \boldsymbol{P}} L_{\boldsymbol{F}} h(\boldsymbol{X}) w\right]
\end{aligned}
$$

which in turn deduces

$$
L_{\Delta F} L_{F} h(X)=L_{\Delta G} L_{F} h(X)=L_{\Delta P} L_{F} h(X)=0
$$

By the definition of relative degree and Eq. (17), Eq. (25) becomes

$$
\dot{z}_{2}=L_{F}^{2} h(X)=z_{3}
$$

After differentiating $r$ times, we find that

$$
\begin{aligned}
& \dot{z}_{r}=L_{G} L_{\boldsymbol{F}}^{r-1} h(\boldsymbol{X}) u+L_{\boldsymbol{F}}^{r} h(\boldsymbol{X})+L_{\Delta \boldsymbol{F}} L_{\boldsymbol{F}}^{r-1} h(\boldsymbol{X})+L_{\Delta \boldsymbol{G}} L_{\boldsymbol{F}}^{r-1} h(\boldsymbol{X}) u+L_{\Delta \boldsymbol{P}} L_{\boldsymbol{F}}^{r-1} h(\boldsymbol{X}) w \\
& =\left[L_{\Delta \boldsymbol{F}} L_{\boldsymbol{F}}^{r-1} h(\boldsymbol{X})+\alpha(\boldsymbol{X}) L_{\Delta \boldsymbol{G}} L_{\boldsymbol{F}}^{r-1} h(\boldsymbol{X})\right]+\left[1+\beta(\boldsymbol{X}) L_{\Delta \boldsymbol{G}} L_{\boldsymbol{F}}^{r-1} h(\boldsymbol{X})\right] v+L_{\Delta \boldsymbol{P}} L_{\boldsymbol{F}}^{r-1} h(\boldsymbol{X}) w
\end{aligned}
$$

Based on the above proof, the disturbance decoupling problem of uncertain affine nonlinear systems can be solved, if there exist VSC matching conditions such that

$$
\begin{gathered}
\text { (c1) } L_{\Delta F} L_{F}^{i} h(\boldsymbol{X})=0, L_{\Delta G} L_{F}^{i} h(\boldsymbol{X})=0, L_{\Delta P} L_{F}^{i} h(\boldsymbol{X})=0, L_{\Delta \boldsymbol{P}} L_{F}^{i} h(\boldsymbol{X})=0,(0 \leq i \leq r-2) \\
\text { (c2) }\|\Delta \boldsymbol{F}(\boldsymbol{X})\| \leq f_{m},\|\Delta \boldsymbol{G}(\boldsymbol{X})\| \leq g_{m},\|\Delta \boldsymbol{P}(\boldsymbol{X})\| \leq p_{m},\|w\| \leq w_{m}
\end{gathered}
$$

where $\|\cdot\|$ is a norm of the vector or matrix of "•", that is $\left\|\left(a_{i j}\right)_{n \times n}\right\|=\max _{1 \leq i \leq n} \sum_{j=1}^{n}\left|a_{i j}\right| ; f_{m}, g_{m}, p_{m}, w_{m}$ are perturbation boundaries of the corresponding given matrixes.

Summing up the definition of the relative degree, matching conditions (c1) and the coordinate transformation $Z=\psi(X)$, we obtain a closed-loop system over the new coordinate by substituting the state feedback (18) or (19) into Eq. (21), which has the form

$$
\begin{gathered}
\dot{z}_{i}=z_{i+1}, \quad 1 \leq i \leq r-1, \\
\dot{z}_{r}=\left[L_{\Delta \boldsymbol{F}} L_{\boldsymbol{F}}^{r-1} h(\boldsymbol{X})+\alpha(\boldsymbol{X}) L_{\Delta \boldsymbol{G}} L_{\boldsymbol{F}}^{r-1} h(\boldsymbol{X})\right]+\left[1+\beta(\boldsymbol{X}) L_{\Delta \boldsymbol{G}} L_{\boldsymbol{F}}^{r-1} h(\boldsymbol{X})\right] v+L_{\Delta \boldsymbol{P}} L_{\boldsymbol{F}}^{r-1} h(\boldsymbol{X}) w \\
\dot{\boldsymbol{\mu}}=\boldsymbol{Q}(\boldsymbol{Z}, \boldsymbol{\mu})+\Delta \boldsymbol{Q}(\boldsymbol{Z}, \boldsymbol{\mu})+\Delta \boldsymbol{R}(\boldsymbol{Z}, \boldsymbol{\mu}) v+[\boldsymbol{K}(\mathbf{Z}, \boldsymbol{\mu})+\Delta \boldsymbol{K}(\boldsymbol{Z}, \boldsymbol{\mu})] w
\end{gathered}
$$


It can be noticed from Eq. (29) that for the state variables $z_{i}$ of the first $r$ - 1 dimensions, the linearization and the disturbance decoupling have been achieved, except for the remaining $z_{r}$ (Eq. (30)). By virtue of the invariance of the sliding mode in VSC [28], it will be used in consequence to eliminate the disturbance and the uncertainty on $z_{r}$.

Based on the VSC theory [28], a switching function is designed easily by taking advantage of the linearized decoupling subsystem (29) over the new coordination

$$
S_{\boldsymbol{Z}}=S(\boldsymbol{Z})=\boldsymbol{C}\left[z_{1} \cdots z_{r}\right]^{T}
$$

where $C=\left[c_{1}, \ldots, c_{r-1}, 1\right]$ is a normal constant coefficient matrix to be determined. Once the system is controlled towards the sliding mode, it satisfies

$$
S_{\boldsymbol{Z}}=\boldsymbol{C}\left[z_{1} \cdots z_{r}\right]^{T}=0
$$

yielding the following reduced-order equation

$$
\dot{z}_{i}=z_{i+1}, \quad 1 \leq i \leq r-1
$$

Clearly, a desired dynamic performance of each state variable in Eq. (33) can be achieved by configuring the coefficient $C$.

As the desired dynamic performance of the sliding mode has already been achieved, an appropriate VSC law is to be defined so as to ensure the desired sliding mode occurring within a finite time. It is convenient to differentiate the switching function (31), and derive the following equation in terms of Eq. (29) :

where

$$
\dot{S}_{\boldsymbol{Z}}=\boldsymbol{A}_{S_{Z}}+\Delta \boldsymbol{A}_{S_{Z}}+\left(\boldsymbol{B}_{S_{Z}}+\Delta \boldsymbol{B}_{S_{Z}}\right) v+\Delta \boldsymbol{C}_{S_{Z}} w
$$

Considering an VSC law below

$$
\begin{gathered}
\boldsymbol{A}_{S_{Z}}=\sum_{i=1}^{r-1} c_{i} \dot{z}_{i}, \Delta \boldsymbol{A}_{S_{Z}}=\boldsymbol{\varphi} \cdot \Delta \boldsymbol{F}+\alpha \boldsymbol{\varphi} \cdot \Delta \boldsymbol{G}, \boldsymbol{B}_{S_{Z}}=1, \Delta \boldsymbol{B}_{S_{Z}}=\beta \boldsymbol{\varphi} \cdot \Delta \boldsymbol{G}, \\
\Delta \boldsymbol{C}_{S_{\boldsymbol{Z}}}=\boldsymbol{\varphi} \cdot \Delta \boldsymbol{P}, \boldsymbol{\varphi}=\frac{\partial L_{\boldsymbol{F}}^{r-1} h(\boldsymbol{X})}{\partial \boldsymbol{X}} .
\end{gathered}
$$

$$
v=-\boldsymbol{B}_{S_{\boldsymbol{Z}}}^{-1}\left[\boldsymbol{A}_{S_{\boldsymbol{Z}}}+a_{s} S_{\boldsymbol{Z}}+b_{s} \operatorname{sgn}\left(S_{\boldsymbol{Z}}\right)\right] \quad\left(a_{s} \geq 0, b_{s}>0\right)
$$

an inequality below can be derived from the matching condition (c2), Eqs. (31) and (34).

$$
\begin{aligned}
& S_{\boldsymbol{Z}} \dot{S}_{\boldsymbol{Z}}=S_{\boldsymbol{Z}}\left\{\Delta \boldsymbol{A}_{S_{\boldsymbol{Z}}}+\Delta \boldsymbol{C}_{S_{\boldsymbol{Z}}} w-a_{s} S_{\boldsymbol{Z}}-b_{s} \operatorname{sgn}\left(S_{\boldsymbol{Z}}\right)-\Delta \boldsymbol{B}_{S_{\boldsymbol{Z}}} \boldsymbol{B}_{S_{\boldsymbol{Z}}}^{-1}\left[\boldsymbol{A}_{S_{\boldsymbol{Z}}}+a_{s} S_{\boldsymbol{Z}}+b_{s} \operatorname{sgn}\left(S_{\boldsymbol{Z}}\right)\right]\right\} \\
& \leq\left\|S_{\boldsymbol{Z}}\right\|\left\|\Delta \boldsymbol{A}_{S_{\boldsymbol{Z}}}\right\|+\left\|S_{\boldsymbol{Z}}\right\|\left\|\Delta \boldsymbol{C}_{S_{\boldsymbol{Z}}} w\right\|-a_{s}\left\|S_{\boldsymbol{Z}}\right\|^{2}-b_{s}\left\|S_{\boldsymbol{Z}}\right\|+\left\|\Delta \boldsymbol{B}_{S_{\boldsymbol{Z}}}\right\|\left(\left\|\boldsymbol{B}_{S_{\boldsymbol{Z}}}^{-1} \boldsymbol{A}_{S_{\boldsymbol{Z}}}\right\|\left\|S_{\boldsymbol{Z}}\right\|+a_{s}\left\|S_{\boldsymbol{Z}}\right\|^{2}+b_{s}\left\|S_{\boldsymbol{Z}}\right\|\right) \\
& \leq\left\|S_{\boldsymbol{Z}}\right\|\|\boldsymbol{\varphi}\|\left(f_{m}-\|\alpha\| g_{m}\right)+\left\|S_{\boldsymbol{Z}}\right\|\|\boldsymbol{\varphi}\| p_{m} w_{m}-a_{s}\left\|S_{\boldsymbol{Z}}\right\|^{2} \\
& -b_{s}\left\|S_{\boldsymbol{Z}}\right\|+\|\beta \boldsymbol{\varphi}\| g_{m}\left(\left\|\boldsymbol{B}_{S_{\boldsymbol{Z}}}^{-1} \boldsymbol{A}_{S_{\boldsymbol{Z}}}\right\|\left\|S_{\boldsymbol{Z}}\right\|+a_{s}\left\|S_{\boldsymbol{Z}}\right\|^{2}+b_{s}\left\|S_{\boldsymbol{Z}}\right\|\right) \\
& =-\left\|S_{\boldsymbol{Z}}\right\|^{2} a_{s}\left(1-\|\beta \boldsymbol{\varphi}\| g_{m}\right)-\left\|S_{\boldsymbol{Z}}\right\|\left\{b_{s}\left(1-\|\beta \boldsymbol{\varphi}\| g_{m}\right)\right. \\
& \left.-\left[\|\varphi\|\left(f_{m}-\|\alpha\| g_{m}+p_{m} w_{m}\right)+\|\beta \boldsymbol{\varphi}\| \boldsymbol{B}_{S_{\boldsymbol{Z}}}^{-1} \boldsymbol{A}_{S_{\boldsymbol{Z}}} \| g_{m}\right]\right\}
\end{aligned}
$$


It is noticed from Eq. (36) that if the perturbation boundary $g_{m}$ of uncertain part $\Delta G$ satisfies

$$
g_{m}<\|\beta \varphi\|^{-1}
$$

then defining

$$
b_{s}>\frac{\|\boldsymbol{\varphi}\|\left(f_{m}-\|\alpha\| g_{m}+p_{m} w_{m}\right)+\|\beta \boldsymbol{\varphi}\|\left\|\boldsymbol{B}_{S_{Z}}^{-1} \boldsymbol{A}_{S_{Z}}\right\| g_{m}}{1-\|\beta \boldsymbol{\varphi}\| g_{m}}
$$

may lead to the following inequality:

$$
S_{Z} \dot{S}_{Z}<0
$$

Namely, the convergence condition of the sliding mode is achieved.

From the above verification, the desired sliding mode is achievable under the VSC law (35), as long as the matching condition (c2) and the constraints (38) are satisfied. Since Eqs. (31) and (35) are the switching function and the control law over the new coordinate $X$, they should be transferred back to the original coordinate $Z$ by adopting the inverse transformation $Z=\boldsymbol{\psi}(X)$. Finally, the DDRC law can be achieved by substituting the VSC law over the original coordinate into the disturbance decoupling state feedback control law (Eq. (18) or Eq. (19)).

To summarize, for an uncertain affine nonlinear system, if the disturbance decoupling condition (17) or (20) and the matching conditions of (c1) and (c2) hold respectively for the certain part and the uncertain part, the DDRC method with the combination of NDD and VSC theories can be figured out as the following design procedure:

Step 1. According to the NDD theory of affine nonlinear systems, the feedback control law (Eq. (18) or (19)) and the coordinate transformation (Eqs. (12) and (13)) are derived to transfer the original system into the linearized decoupling normal form (Eq. (15)) over the new coordinate.

Step 2. Give the VSC matching conditions (c1) and (c2) for the uncertain part of the affine nonlinear systems.

Step 3. Utilize the linearized decoupling normal form (Eq. (15)) over the new coordinate to design the switching function (Eq. (31)), and determine its coefficients accordingly.

Step 4. Design the VSC law (Eq. (35)) based on the perturbation boundary (37) of the uncertainty part, and the convergence condition of the sliding mode (39).

Step 5. Define the coordinate transformation (12) to transfer the switching function (Eq. (31)) and the VSC law (Eq. (35)) from the new coordinate $Z$ back to the original coordinate $X$.

Step 6. Substitute the VSC law (Eq. (18) or (19)) over the original coordinate into the feedback control law, and yield the DDRC method.

A block diagram of the closed-loop system for the aforementioned DDRC method is shown in Figure 8, which includes two feedback loops. The nonlinear loop (i.e., the NDD loop) is used to achieve the disturbance decoupling and the partial linearization, regarding the system output $y$ from the disturbance $w$. On the other hand, the linear loop (i.e., the VSC loop) is used to restrain the system's uncertainty and regulate the closed-loop dynamic performance. 


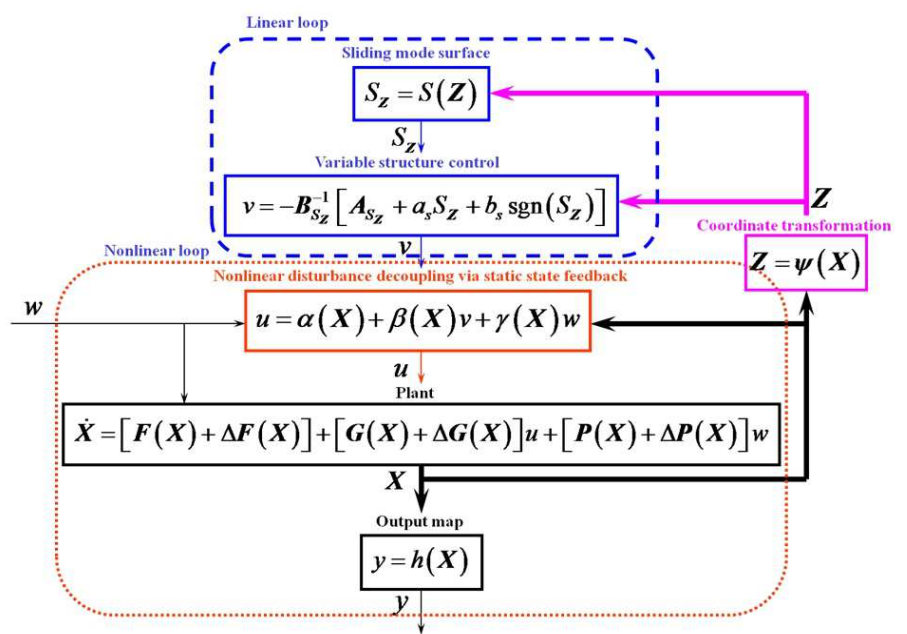

Fig. 8. Block diagram of closed-loop system for DDRC method

\section{LFS AACC system}

In this section, the proposed DDRC method will be used to design the LFS AACC system with respect to the driving and the braking conditions.

\subsection{LFS AACC system for driving condition}

Recall the procedure in 2.2, the disturbance decoupling problem on the LFS dynamics model without the impact of the uncertainty is considered (i.e., for the uncertain items of Eq. (7) let $\left.\Delta \boldsymbol{F}_{a}(X)=0, \Delta \boldsymbol{G}_{a}(\boldsymbol{X})=0\right)$. On the purpose of LFS AACC system, the following affine nonlinear system with the output variable is defined:

$$
\left\{\begin{array}{l}
\dot{\boldsymbol{X}}=\boldsymbol{F}_{a}(\boldsymbol{X})+\boldsymbol{G}_{a}(\boldsymbol{X}) \alpha_{t h}+\boldsymbol{P}_{a}(\boldsymbol{X}) w \\
y=h(\boldsymbol{X})=\varepsilon_{d}
\end{array}\right.
$$

By adopting the NDD theory of certain affine nonlinear system, the relative degree of system (40) is calculated as

$$
L_{F_{a}} h(X)=\varepsilon_{v}, L_{G_{a}} h(X)=\left[\begin{array}{llll}
1 & 0 & 0 & 0
\end{array}\right] G_{a}=0, L_{G_{a}} L_{F_{a}} h(X)=\left[\begin{array}{llll}
0 & 1 & 0 & 0
\end{array}\right] G_{a} \neq 0 .
$$

Obviously, the relative degree is $r=2$, which results in the following matrix

$$
\Omega_{a}(\boldsymbol{X})=\left[\begin{array}{c}
d h(X) \\
d L_{F_{a}} h(\boldsymbol{X})
\end{array}\right]=\left[\begin{array}{llll}
1 & 0 & 0 & 0 \\
0 & 1 & 0 & 0
\end{array}\right]
$$

Then, it is easy to verify that

$$
\Omega_{a}(\boldsymbol{X}) \boldsymbol{P}_{a}=\left[\begin{array}{llll}
1 & 0 & 0 & 0 \\
0 & 1 & 0 & 0
\end{array}\right] \boldsymbol{P}_{a}=\left[\begin{array}{c}
0 \\
p_{a 1}
\end{array}\right] \neq \mathbf{0}
$$


That is to say, the disturbance decoupling from system (40) can not be achieved by the state feedback (18), because the necessary and sufficient condition (17) is not satisfied. Thus, one can turn to the state feedback (19) with measurable disturbance. Note that if

$$
\gamma_{a}=-\left(\frac{p_{a 1}}{g_{a 1}}\right)
$$

then the necessary and sufficient condition (20) is satisfied, i.e.,

$$
\Omega_{a}(X)\left[G_{a} \gamma_{a}+P_{a}\right]=\left[\begin{array}{llll}
1 & 0 & 0 & 0 \\
0 & 1 & 0 & 0
\end{array}\right]\left[G_{a} \gamma_{a}+P_{a}\right]=\mathbf{0}
$$

By Eq. (19), the decoupling state feedback is obtained as

$$
\alpha_{t h}=\alpha_{a}(\boldsymbol{X})+\beta_{a}(\boldsymbol{X}) v_{u a}+\gamma_{a}(\boldsymbol{X}) w=\frac{-f_{a 2}\left(\omega_{t}, \omega_{e d}\right)+v_{u a}-p_{a 1} w}{g_{a 1}\left(\omega_{t}, \omega_{e d}\right)}
$$

and the corresponding coordinate transformation with $r=2$ dimensions is

$$
\boldsymbol{Z}_{a}=\boldsymbol{\psi}_{a}(\boldsymbol{X})=\left[\begin{array}{c}
z_{a 1} \\
z_{a 2}
\end{array}\right]=\left[\begin{array}{c}
h(\boldsymbol{X}) \\
L_{\boldsymbol{F}_{a}} h(\boldsymbol{X})
\end{array}\right]=\left[\begin{array}{c}
\varepsilon_{d} \\
\varepsilon_{v}
\end{array}\right]
$$

where

$$
L_{F_{a}}^{2} h(X)=f_{a 2}, L_{P_{a}} L_{F_{a}} h(X)=p_{a 1}, L_{G_{a}} L_{F_{a}} h(X)=g_{a 1} .
$$

Additionally, in order to complete the coordinate transformation, the remaining $n-r=2$ dimensional coordinates $\mu_{a 1}, \mu_{a 2}$ should satisfy the following condition:

$$
\frac{\partial \mu_{a i}}{\partial \boldsymbol{X}} G_{a}=\left[\begin{array}{llll}
\frac{\partial \mu_{a i}}{\partial \varepsilon_{d}} & \frac{\partial \mu_{a i}}{\partial \varepsilon_{v}} & \frac{\partial \mu_{a i}}{\partial \omega_{t}} & \frac{\partial \mu_{a i}}{\partial \omega_{e d}}
\end{array}\right]\left[\begin{array}{c}
0 \\
g_{a 1} \\
0 \\
g_{a 2}
\end{array}\right]=0 \quad(i=1,2)
$$

The purpose is to ensure the diffeomorphism relationship of the coordinate transformation between the original and the new one (in other words, it is a one-to-one continuous coordinate transformation between the original and the new one, the same is for the inverse transformation). Obviously, one solution of the partial differential Eq. (47) is

$$
\begin{aligned}
& \mu_{a 1}=\omega_{t} \\
& \mu_{a 2}=-\varepsilon_{v}+\alpha_{n} t_{h}\left(b \omega_{t} \omega_{e d}+c \omega_{e d}^{2}+d \frac{\omega_{t}^{3}}{\omega_{e d}}\right)
\end{aligned}
$$

Hence, the transformation of the remaining 2 dimensional coordinates is

$$
\boldsymbol{\mu}_{a}=\boldsymbol{\phi}_{a}(\boldsymbol{X})=\left[\begin{array}{l}
\mu_{a 1}(\boldsymbol{X}) \\
\mu_{a 2}(X)
\end{array}\right]
$$


Up to now, the decoupling state feedback (Eq. (45)) and the coordinate transformation (Eqs. (46) and (49)) have been obtained for the certain part of the LFS dynamics model under the driving condition.

Further consideration on the uncertain part of model (7) will be continued. On the basis of the design procedure (Step2) in 3.2, the matching conditions (c1) and (c2) have to be verified at first, and

$$
\begin{aligned}
& L_{\Delta \boldsymbol{F}_{a}} h(\boldsymbol{X})=\left[\begin{array}{llll}
1 & 0 & 0 & 0
\end{array}\right] \Delta \boldsymbol{F}_{a}=0 \\
& L_{\Delta \boldsymbol{G}_{a}} h(\boldsymbol{X})=\left[\begin{array}{lllll}
1 & 0 & 0 & 0 & 0
\end{array}\right] \Delta \boldsymbol{G}_{a}=0
\end{aligned}
$$

It should be noticed from 1.2 and 1.3 that the uncertain items $\Delta \boldsymbol{F}_{a}(\boldsymbol{X}), \Delta \boldsymbol{G}_{a}(\boldsymbol{X})$ and the disturbance $w$ are subject to the following limited upper boundaries:

$$
\begin{aligned}
& \left\|\Delta F_{a}(X)\right\| \leq f_{a m}=203 \\
& \left\|\Delta G_{a}(X)\right\| \leq g_{a m}=0.031 \\
& \|w\| \leq w_{a m}=2
\end{aligned}
$$

By substituting the decoupling state feedback $u=a_{\text {th }}$ (Eq. (45)) into model (7), and making use of the coordinate transformations (46) and (49), a linearized subsystem below can be achieved, in which the certain part is completely decoupled from the disturbance.

$$
\{\left[\begin{array}{c}
\dot{z}_{a 1} \\
\dot{z}_{a 2}
\end{array}\right]=\overbrace{\left[\begin{array}{cc}
0 & 1 \\
0 & 0
\end{array}\right]\left[\begin{array}{l}
z_{a 1} \\
z_{a 2}
\end{array}\right]+\left[\begin{array}{l}
0 \\
1
\end{array}\right] v_{u a}}^{\text {Certain part }}+\overbrace{\left[\begin{array}{c}
0 \\
\Delta f_{a 2}-\frac{f_{a 2}}{g_{a 1}} \Delta g_{a 1} \\
y=\varepsilon_{d}
\end{array}\right]+\left[\begin{array}{c}
0 \\
\frac{\Delta g_{a 1}}{g_{a 1}}
\end{array}\right] v_{u a}+\left[\begin{array}{c}
0 \\
-\frac{p_{a 1}}{g_{a 1}} \Delta g_{a 1}
\end{array}\right] w}^{\text {Part of uncertain and disturbance }}
$$

Besides, a nonlinear dynamic internal subsystem without separating from the disturbance and the uncertainty is yielded

where

$$
\dot{\boldsymbol{\mu}}_{a}=\boldsymbol{Q}_{a}\left(\boldsymbol{Z}_{a}, \boldsymbol{\mu}_{a}\right)+\Delta \boldsymbol{Q}_{a}\left(\boldsymbol{Z}_{a}, \boldsymbol{\mu}_{a}\right)+\left[\boldsymbol{K}_{a}\left(\boldsymbol{Z}_{a}, \boldsymbol{\mu}_{a}\right)+\Delta \boldsymbol{K}_{a}\left(\boldsymbol{Z}_{a}, \boldsymbol{\mu}_{a}\right)\right] w
$$

$$
\boldsymbol{Q}_{a}\left(\boldsymbol{Z}_{a}, \boldsymbol{\mu}_{a}\right)=\left[\begin{array}{c}
a \mu_{a 1}^{2}+\frac{\mu_{a 2}+z_{a 2}}{\alpha_{n} t_{h}}+l \\
-\left(2 a \mu_{a 1}+\frac{1}{t_{h}}\right)\left(\alpha_{n} t_{h} a \mu_{a 1}^{2}+\mu_{a 2}+z_{a 2}+\alpha_{n} t_{h} l\right)
\end{array}\right], \boldsymbol{K}_{a}\left(\mathbf{Z}_{a}, \boldsymbol{\mu}_{a}\right)=\left[\begin{array}{c}
0 \\
-p_{a 1}
\end{array}\right]
$$

Based on the analysis of the extreme operating conditions in 2.1, it can be noticed that the items $\Delta \boldsymbol{Q}_{\mathrm{a}}, \Delta \boldsymbol{K}_{\mathrm{a}}$ are constants with limited upper boundaries.

For the certain part of Eq. (52), it is clear that the state variables $z_{a 1}, z_{a 2}$ have been completely decoupled from the disturbance $w$. In order to enhance the system's robustness from the remaining uncertain part and the disturbance within the linearized decoupling subsystem (52), we may design the following switching function over the new coordinate by making use of Eq. (52). 


$$
S_{a Z}=\boldsymbol{C}_{a}\left[\begin{array}{l}
z_{a 1} \\
z_{a 2}
\end{array}\right]
$$

where $C_{a}=\left[\begin{array}{ll}C_{a 1} & 1\end{array}\right]$ is a coefficient matrix to be determined. Once the system is controlled towards the sliding mode, it obeys

$$
S_{a Z}=c_{a 1} z_{a 1}+z_{a 2}=0 \Rightarrow z_{a 2}=-c_{a 1} z_{a 1}
$$

and the order of Eq. (52) can be reduced to

$$
\dot{z}_{a 1}=z_{a 2}
$$

Clearly, the disturbance and the uncertainty have been separated from Eq. (56). In this way, substituting Eq. (56) into Eq. (55) yields

$$
\dot{z}_{a 1}+c_{a 1} z_{a 1}=0
$$

By the Laplace transform, an eigenvalue equation of Eq. (57) is obtained as

$$
s+c_{a 1}=0
$$

To achieve a desired dynamic performance and a stable convergence of the sliding mode, the coefficient $c_{a 1}$ can be determined by employing the pole assignment method. That is, the eigenvalue of Eq. (58) should be assigned strictly in the negative half plane. Without loss of generality, it can be chosen herein as $c_{a 1}=1$.

The VSC law is designed below by the procedure (Step4) of 3.2, in order to guarantee that the desired sliding mode occurs within a finite time. First, a VSC law is obtained on the basis of Eq. (35):

$$
v_{u a}=-\boldsymbol{B}_{S_{a Z}}^{-1}\left[\boldsymbol{A}_{S_{a Z}}+a_{a s} S_{a \boldsymbol{Z}}+b_{a s} \operatorname{sgn}\left(S_{a \boldsymbol{Z}}\right)\right]
$$

where $\boldsymbol{A}_{S_{a Z}}=c_{a 1} \dot{z}_{a 1}, \boldsymbol{B}_{S_{a Z}}=1$. For determining the coefficients $a_{a s}, b_{a s}$, the perturbation boundary of $g_{a m}$ should be verified such that

$$
g_{a m}<\left\|\beta_{a} \boldsymbol{\varphi}_{a}\right\|^{-1}
$$

where $\boldsymbol{\varphi}_{a}=\left[\begin{array}{lll}0 & 1 & 0\end{array}\right]$. According to Eq. (45) and the analysis of 3.2, it is easy to obtain

$$
\left\|\beta_{a} \boldsymbol{\varphi}_{a}\right\|^{-1}=\left(\max \left|\frac{1}{g_{a 1}\left(\omega_{t}, \omega_{e d}\right)}\right|\right)^{-1}=0.98
$$

Clearly, the condition of Eq. (60) is satisfied. Then, the parameter $b_{a s}$ will be determined by the inequality (38). Recalling the analysis results of 3.1, $\left\|\alpha_{a}\right\|=\max \left|-\frac{f_{a 2}\left(\omega_{t}, \omega_{e d}\right)}{g_{a 1}\left(\omega_{t}, \omega_{e d}\right)}\right|=16.33$ is given. On this basis, it is reasonable to suppose that the absolute value of the extreme relative velocity tracking error is $\max \left|\varepsilon_{v}\right|=35 \mathrm{~m} / \mathrm{s}$. It can be presented as a scenario that the leading vehicle moves forward with a maximum velocity $35 \mathrm{~m} / \mathrm{s}$ relative to the statical 
controlled vehicle (assuming this given value is an actual maximum velocity). The values above will be substituted into the right hand side of the inequality (38), and we have

$$
\frac{\left\|\boldsymbol{\varphi}_{a}\right\|\left(f_{a m}-\left\|\alpha_{a}\right\| g_{a m}\right)+\left\|\beta_{a} \boldsymbol{\varphi}_{a}\right\|\left\|\boldsymbol{B}_{S_{a Z}}^{-1} \boldsymbol{A}_{S_{a Z}}\right\| g_{a m}}{1-\left\|\beta_{a} \boldsymbol{\varphi}_{a}\right\| g_{a m}}=210.25
$$

Then, the parameter $b_{a s}=250$ can be determined, and $a_{a s}=10$ is achieved separately by the condition of $a_{a s}>0$.

By the procedure (Step5) in 3.2, the coordinate transformations $Z_{a}=\boldsymbol{\psi}_{a}(\boldsymbol{X})$ and $\boldsymbol{\mu}_{a}=\boldsymbol{\phi}_{a}(\boldsymbol{X})$ will be used to transfer the new coordinates $\left(Z_{a}, \boldsymbol{\mu}_{a}\right)$ back to the original coordinate $X$. In this way, the switching function over the original coordinate becomes

$$
S_{a \boldsymbol{Z}}=c_{a 1} z_{a 1}+z_{a 2} \stackrel{Z_{a}=\boldsymbol{\psi}_{a}(X)}{\Rightarrow} S_{a \boldsymbol{X}}=c_{a 1} \varepsilon_{d}+\varepsilon_{v}
$$

the VSC law (57) over the original coordinate has the form

$$
v_{u a}=-\left[c_{a 1} \varepsilon_{v}+a_{a s} S_{a X}+b_{a s} \operatorname{sgn}\left(S_{a X}\right)\right]
$$

With substitutions of $S_{a X}$ and $v_{u a}$ into Eq. (45), a AACC system based on the DDRC method is finally obtained as

$$
\alpha_{t h}=\frac{-f_{a 2}\left(\omega_{t}, \omega_{e d}\right)}{g_{a 1}\left(\omega_{t}, \omega_{e d}\right)}-\frac{\left[c_{a 1} \varepsilon_{v}+a_{a s}\left(c_{a 1} \varepsilon_{d}+\varepsilon_{v}\right)+b_{a s} \operatorname{sgn}\left(S_{a X}\right)\right]+p_{a 1} w}{g_{a 1}\left(\omega_{t}, \omega_{e d}\right)}
$$

The control laws designed above only satisfy the convergence stability and the robustness of the linearized decoupling subsystem. In order to ensure the stability of the total system, the stability of the remaining nonlinear internal dynamic subsystem has to be verified, so that the problem of tracking control can be solved completely. Based on ref. [38], the study on the stability of nonlinear internal dynamic subsystem can be turned into the study on its zero dynamics correspondingly. Therefore, let $\Delta Q_{\mathrm{a}}=\Delta K_{\mathrm{a}}=0$, i.e., ignore the tiny impact of the uncertain part. Then the zero dynamics of the nonlinear internal dynamic subsystem (53) owing to $z_{a 1}, z_{a 2}, w=0$ is obtained as follows

$$
\left\{\begin{array}{l}
\dot{\mu}_{a 1}=a \mu_{a 1}^{2}+\frac{\mu_{a 2}}{\alpha_{n} t_{h}}+l \\
\dot{\mu}_{a 2}=-\left(2 a \mu_{a 1}+\frac{1}{t_{h}}\right)\left(\alpha_{n} t_{h} a \mu_{a 1}^{2}+\mu_{a 2}+\alpha_{n} t_{h} l\right)
\end{array}\right.
$$

To verify the asymptotic stability of Eq. (66) at the equilibrium point $\left(z_{a 1}, z_{a 2}, \mu_{a 1}, \mu_{a 2}\right)=0$, a candidate Lyapunov function is chosen:

$$
V_{a}\left(\mu_{a 1}, \mu_{a 2}\right)=-\left(\alpha_{n} \mu_{a 1}+\mu_{a 2}\right)^{2}
$$

The time derivative with respect to the Lyapunov function Eq. (67) is 


$$
\begin{aligned}
& \frac{d V_{a}}{d t}=-2\left(\alpha_{n} \mu_{a 1}+\mu_{a 2}\right)\left(\alpha_{n} \dot{\mu}_{a}+\dot{\mu}_{a}\right) \\
& =2 a \mu_{a 1}\left(\alpha_{n} t_{h} a \mu_{a 1}^{2}+\mu_{a 2}+\alpha_{n} t_{h} l\right) \times\left[\alpha_{n} \omega_{t}+\alpha_{n} t_{h}\left(b \omega_{t} \omega_{e d}+c \omega_{e d}^{2}+d \frac{\omega_{t}^{3}}{\omega_{e d}}\right)\right]
\end{aligned}
$$

Because $a, b, c, d, \alpha_{n}, t_{h}, l, \omega_{t}, \omega_{e d}>0$ and $\dot{\omega}_{t}>0$ under the driving condition of the vehicle acceleration, we have

$$
\alpha_{n} t_{h} a \mu_{a 1}^{2}+\mu_{a 2}+\alpha_{n} t_{h} l=\alpha_{n} t_{h} \dot{\mu}_{a 1}=\alpha_{n} t_{h} \dot{\omega}_{t}>0,\left(\text { if } \dot{\omega}_{t} \neq 0\right)
$$

In addition, it is easy to verify

$$
\alpha_{n} \omega_{t}+\alpha_{n} t_{h}\left(b \omega_{t} \omega_{e d}+c \omega_{e d}^{2}+d \frac{\omega_{t}^{3}}{\omega_{e d}}\right)>0 \quad\left(\text { if } \omega_{t} \neq \omega_{e d} \neq 0\right)
$$

Therefore, $\frac{d V_{a}}{d t}>0$. The following inequality is satisfied:

$$
V_{a} \frac{d V_{a}}{d t}<0 \quad\left(\text { is } \omega_{t} \neq \omega_{e d} \neq 0 \text { and } \dot{\omega}_{t} \neq 0\right)
$$

The zero dynamics is asymptotically stable.

\subsection{LFS AACC system for braking condition}

The design of LFS AACC system under the braking condition is similar to under the driving condition. Regarding the purpose of the LFS AACC system (8), the output can be defined as $y=h(X)=\varepsilon_{d}$. Then, the relative degree is obtained as $r=2$, and the decoupling state feedback is achieved according to Eq. (19) as

$$
u_{b}=\alpha_{d}(X)+\beta_{d}(X) v_{u d}+\gamma_{d}(X) w=\frac{-f_{d 2}\left(\omega_{t}, \omega_{e d}, a_{b}\right)+v_{u d}-p_{d 1} w}{g_{d 1}\left(\omega_{t}, a_{b}\right)}
$$

The corresponding coordinate transformation is given as

$$
\begin{aligned}
& \boldsymbol{Z}_{d}=\boldsymbol{\psi}_{d}(\boldsymbol{X})=\left[\begin{array}{l}
z_{d 1} \\
z_{d 2}
\end{array}\right]=\left[\begin{array}{c}
h(X) \\
L_{\boldsymbol{F}_{d}} h(\boldsymbol{X})
\end{array}\right]=\left[\begin{array}{l}
\varepsilon_{d} \\
\varepsilon_{v}
\end{array}\right] \\
& \boldsymbol{\mu}_{d}=\boldsymbol{\phi}_{d}(\boldsymbol{X})=\left[\begin{array}{c}
\mu_{d 1}(\boldsymbol{X}) \\
\mu_{d 2}(\boldsymbol{X}) \\
\mu_{d 3}(\boldsymbol{X})
\end{array}\right]=\left[\begin{array}{c}
\omega_{t} \\
\omega_{e d} \\
\varepsilon_{v}-\alpha_{n} t_{h} d_{d} a_{b}
\end{array}\right]
\end{aligned}
$$

Taking further account of the influence from system's uncertainty, we have

$$
\begin{aligned}
& L_{\Delta \boldsymbol{F}_{d}} h(\boldsymbol{X})=\left[\begin{array}{llll}
1 & 0 & 0 & 0
\end{array}\right] \Delta \boldsymbol{F}_{d}=0 \\
& L_{\Delta \boldsymbol{G}_{d}} h(\boldsymbol{X})=\left[\begin{array}{lllll}
1 & 0 & 0 & 0 & 0
\end{array}\right] \Delta \boldsymbol{G}_{d}=0
\end{aligned}
$$


That is to say, the matching condition (c1) is satisfied with respect of uncertain items $\Delta \boldsymbol{F}_{d}(\boldsymbol{X})$, $\Delta G_{d}(X)$. Besides, on the analyses of 2.2 and 2.3, the uncertain items $\Delta \boldsymbol{F}_{d}(\boldsymbol{X}), \Delta \boldsymbol{G}_{d}(\boldsymbol{X})$ and the disturbance $w$ are subject to the following limited upper boundaries:

$$
\begin{aligned}
& \left\|\Delta \boldsymbol{F}_{d}(\boldsymbol{X})\right\| \leq f_{d m}=192 \\
& \left\|\Delta \boldsymbol{G}_{d}(\boldsymbol{X})\right\| \leq g_{d m}=0.029
\end{aligned}
$$

By substituting the decoupling state feedback (72) into model (8), and making use of the coordinate transformations (73) and (74), a linearized subsystem (77) can be achieved, in which the certain part is completely decoupled from the disturbance.

$$
\{\left[\begin{array}{c}
\dot{z}_{d 1} \\
\dot{z}_{d 2}
\end{array}\right]=\overbrace{\left[\begin{array}{ll}
0 & 1 \\
0 & 0
\end{array}\right]\left[\begin{array}{l}
z_{d 1} \\
z_{d 2}
\end{array}\right]+\left[\begin{array}{l}
0 \\
1
\end{array}\right] v_{u d}}^{\text {Certain part }}+\overbrace{\left[\begin{array}{c}
0 \\
\Delta f_{d 2}-\frac{f_{d 2}}{g_{d 1}} \Delta g_{d 1} \\
y=\varepsilon_{d}
\end{array}\right]+\left[\begin{array}{c}
0 \\
\frac{\Delta g_{d 1}}{g_{d 1}}
\end{array}\right] v_{u d}+\left[\begin{array}{c}
0 \\
-\frac{p_{d 1}}{g_{d 1}} \Delta g_{d 1}
\end{array}\right] w}^{\text {Part of uncertain and disturbance }}
$$

Additionally, a nonlinear internal dynamic subsystem with the influence of the disturbance and uncertainty is presented

$$
\dot{\boldsymbol{\mu}}_{d}=\boldsymbol{Q}_{d}\left(\boldsymbol{Z}_{d}, \boldsymbol{\mu}_{d}\right)+\Delta \boldsymbol{Q}_{d}\left(\mathbf{Z}_{d}, \boldsymbol{\mu}_{d}\right)+\left[\boldsymbol{K}_{d}\left(\boldsymbol{Z}_{d}, \boldsymbol{\mu}_{d}\right)+\Delta \boldsymbol{K}_{d}\left(\boldsymbol{Z}_{d}, \boldsymbol{\mu}_{d}\right)\right] w
$$

where

$$
\boldsymbol{Q}_{d}\left(\mathbf{Z}_{d}, \boldsymbol{\mu}_{d}\right)=\left[\begin{array}{c}
a_{d} \mu_{d 1}^{2}+b_{d} \mu_{d 1} \mu_{d 2}+c_{d} \mu_{d 2}^{2}+\frac{-\mu_{d 3}+z_{d 2}}{\alpha_{n} t_{h}}-\vartheta_{d} \\
e_{d} \mu_{d 1}^{2}+f_{d} \mu_{d 1} \mu_{d 2}+g_{d} \mu_{d 2}^{2}+h_{d} \mu_{d 2}+i_{d} \\
-\left(2 \frac{a_{d}}{d_{d}} \mu_{d 1}+\frac{b_{d}}{d_{d}} \mu_{d 2}+\frac{1}{t_{h} d_{d}}\right) \times\left(a_{d} \mu_{d 1}^{2}+b_{d} \mu_{d 1} \mu_{d 2}+c_{d} \mu_{d 2}^{2}+\frac{-\mu_{d 3}+z_{d 2}}{\alpha_{n} t_{h}}-\vartheta_{d}\right) \\
-\left(\frac{b_{d}}{d_{d}} \mu_{d 1}+2 \frac{c_{d}}{d_{d}} \mu_{d 2}\right) \times\left(e_{d} \mu_{d 1}^{2}+f_{d} \mu_{d 1} \mu_{d 2}+g_{d} \mu_{d 2}^{2}+h_{d} \mu_{d 2}+i_{d}\right)
\end{array}\right]
$$

According to the analysis of 2.1, items $\Delta Q_{d}, \Delta K_{d}$ are the constants with limited upper boundaries.

Similarly, the VSC law can be designed as

$$
v_{u d}=-\boldsymbol{B}_{S_{d \boldsymbol{Z}}}^{-1}\left[\boldsymbol{A}_{S_{d \boldsymbol{Z}}}+a_{d s} S_{d \boldsymbol{Z}}+b_{d s} \operatorname{sgn}\left(S_{d \boldsymbol{Z}}\right)\right]
$$

where the sliding mode surface is

$$
S_{d Z}=c_{d 1} z_{d 1}+z_{d 2}
$$


By ignoring the tedious calculation process, the parameters are given directly as $\boldsymbol{A}_{S_{d Z}}=c_{d 1} \dot{z}_{d 1}, \boldsymbol{B}_{S_{d Z}}=1, c_{d 1}=1, a_{d s}=10, b_{d s}=185$. By transferring $v_{u d}$ back to the original coordinate and substituting it into Eq. (72), the AACC law is finally obtained as

$$
u_{b}=-\frac{f_{d 2}\left(\omega_{t}, \omega_{e d}, a_{b}\right)}{g_{d 1}\left(\omega_{t}, a_{b}\right)}-\frac{\left[c_{d 1} \varepsilon_{v}+a_{d s}\left(c_{d 1} \varepsilon_{d}+\varepsilon_{v}\right)+b_{d s} \operatorname{sgn}\left(S_{d X}\right)\right]+p_{d 1} w}{g_{d 1}\left(\omega_{t}, a_{b}\right)}
$$

where $S_{a X}=c_{d 1} \varepsilon_{d}+\varepsilon_{v}$, which is a sliding mode surface over the original coordinate.

The remaining nonlinear internal dynamic subsystem (78) should be verified as well to ensure the stability of the total system. At first, if $z_{d 1}, z_{d 2}, w=0$ and the impact of uncertain items $\Delta \boldsymbol{Q}_{d}, \Delta \boldsymbol{K}_{d}$ can be neglected, then the zero dynamics becomes

$$
\left\{\begin{aligned}
\dot{\mu}_{d 1}= & a_{d} \mu_{d 1}^{2}+b_{d} \mu_{d 1} \mu_{d 2}+c_{d} \mu_{d 2}^{2}-\frac{\mu_{d 3}}{\alpha_{n} t_{h}}-\vartheta_{d} \\
\dot{\mu}_{d 2}= & e_{d} \mu_{d 1}^{2}+f_{d} \mu_{d 1} \mu_{d 2}+g_{d} \mu_{d 2}^{2}+h_{d} \mu_{d 2}+i_{d} \\
\dot{\mu}_{d 3}= & -\left(2 \frac{a_{d}}{d_{d}} \mu_{d 1}+\frac{b_{d}}{d_{d}} \mu_{d 2}+\frac{1}{t_{h} d_{d}}\right) \times\left(a_{d} \mu_{d 1}^{2}+b_{d} \mu_{d 1} \mu_{d 2}+c_{d} \mu_{d 2}^{2}-\frac{\mu_{d 3}}{\alpha_{n} t_{h}}-\vartheta_{d}\right) \\
& -\left(\frac{b_{d}}{d_{d}} \mu_{d 1}+2 \frac{c_{d}}{d_{d}} \mu_{d 2}\right) \times\left(e_{d} \mu_{d 1}^{2}+f_{d} \mu_{d 1} \mu_{d 2}+g_{d} \mu_{d 2}^{2}+h_{d} \mu_{d 2}+i_{d}\right)
\end{aligned}\right.
$$

Then, a candidate Lyapunov function is chosen as

$$
V_{d}\left(\mu_{d 1}, \mu_{d 2}, \mu_{d 3}\right)=\mu_{d 2}+\left(\frac{2 \pi \alpha_{n} t_{h} d_{d} r_{t}}{60 i_{g} i_{0}} \mu_{d 1}+\int \mu_{d 3} d t\right)
$$

Since $z_{d 2}=0$, it is easy to obtain

$$
V_{d}\left(\mu_{d 1}, \mu_{d 2}, \mu_{d 3}\right)=\mu_{d 2}=\omega_{e d}>0 \quad\left(\text { if } \omega_{e d} \neq 0\right)
$$

The time derivative with respect to the Lyapunov function Eq. (83) is

$$
\frac{d V_{d}}{d t}=\dot{\mu}_{d 2}+\left(\frac{2 \pi \alpha_{n} t_{h} d_{d} r_{t}}{60 i_{g} i_{0}} \dot{\mu}_{d 1}+\mu_{d 3}\right)=\dot{\mu}_{d 2}=\dot{\omega}_{e d}
$$

For the braking condition, the engine operates under the decelerating mode, hence

$$
\frac{d V_{d}}{d t}=\dot{\omega}_{e d}<0\left(\text { if } \dot{\omega}_{e d} \neq 0\right)
$$

Assembling Eqs. (84) and (85), the following inequality is hold

$$
V_{d} \frac{d V_{d}}{d t}<0 \quad\left(\text { if } \omega_{e d} \neq 0 \text { and } \dot{\omega}_{e d} \neq 0\right)
$$

Thus, the zero dynamics of the nonlinear internal dynamic subsystem (78) is asymptotically stable as long as $z_{d 1}, z_{d 2}, w=0$. 


\section{Simulation and analysis}

Base on above analysis of the control system under the driving/braking conditions, the LFS AACC system applying the DDRC method can be designed as the block diagram in Figure 9. The system consists of three parts: the controlled object of a convoy with two vehicles, DDRC system, and the input/output signals.

In order to verify the control performance of the LFS AACC system, a typical driving cycle of the leading vehicle's aceeleration/deceleration, velocity, as well as the road grade are given in Figure 10. The road grade changes from $0 \mathrm{o} \sim+3 \mathrm{o}$ to $0 \mathrm{o} \sim-3 \mathrm{o}$ in a period of $80 \mathrm{~s} \sim 90 \mathrm{~s}$ and $110 \mathrm{~s} \sim 120 \mathrm{~s}$, respectively. Furthermore, the conditions from the high-speed to low-speed SG, and two cases of mass equaling $10,000 \mathrm{~kg}$ and $25,000 \mathrm{~kg}$ are included. The initial errors at $0 \mathrm{~s}$ for the inter-vehicles relative distance and relative velocity are set to $0 \mathrm{~m}$ and $0 \mathrm{~m} / \mathrm{s}$, respectively. Table 1 and the solid lines in Figures 11 and 12 are the coefficients and the simulation results, respectively for the proposed control system. In contrast, the coefficients and some simulation results of an upper LQ+lower PID hierarchical control system proposed in ref. [1] are also presented respectively in Table 2 and by the dotted lines in Figures 11 and 12. The comparison results of the throttle angle, desired input voltage of EBS, engine speed, automatic transmission gear position, relations of relative distance/relative velocity tracking error verses time scale, as well as the phase chart of the relative distance/relative velocity tracking error are shown in Figures 11 and 12 in sequences of (a) (f).

\begin{tabular}{|l|l|l|l|}
\hline Driving condition & $c_{a 1}=1$ & $a_{a \mathrm{~s}}=10$ & $b_{a \mathrm{~s}}=250$ \\
\hline Braking condition & $c_{d 1}=1$ & $a_{d \mathrm{~s}}=10$ & $b_{d \mathrm{~s}}=185$ \\
\hline
\end{tabular}

Table 1. Control parameters of DDRC system

\begin{tabular}{|l|l|l|l|}
\hline \multirow{2}{*}{ Conditions } & \multicolumn{2}{|c|}{$\begin{array}{c}\text { Upper layer LQ } \\
\text { parameters }\end{array}$} & \multicolumn{1}{|c|}{$\begin{array}{c}\text { Lower layer PID } \\
\text { parameters }\end{array}$} \\
\cline { 2 - 4 } & $\mathrm{Q}$ & $\mathrm{R}$ & $\mathrm{P}, \mathrm{I}, \mathrm{D}$ \\
\hline Driving & {$[70,04]$} & \multirow{2}{*}{10} & $800,560,15$ \\
\cline { 4 - 4 } Braking & & & $350,150,20$ \\
\hline
\end{tabular}

Table 2. Control parameters of hierarchical control system

As illustrated by Figures 11 (a) (d), for the proposed control system, the throttle angle and the EBS desired input voltage exhibit smooth response characteristic, rapid convergence and small oscillation, even at the moment of gear switching. However, for the hierarchical control system, it shows intense and long time oscillations especially at low-speed condition (shown as dashed border subfigures inside the Figures 11 (a) and (b)), which have impacts on the vehicle's comfortability severely.

This is because the small parameters are adopted by the proposed control system as the consequence of applying DDRC method (shown as Tables 1), and thus the unmodeled high frequency oscillation can be effectively eliminated, in contrast with the hierarchical control system adopting large parameters (shown as Tables 2). Moreover, during the time period of $0 \mathrm{~s} \sim 73 \mathrm{~s}$ and 130s $\sim 200 \mathrm{~s}$ in Figures 11(e) and 12(e), the simulation results of the proposed control system indicate that the errors of the relative distance and the relative velocity are 
constrained within the range of $\pm 0.02 \mathrm{~m}$ and $-0.05 \mathrm{~m} / \mathrm{s} \sim 0.02 \mathrm{~m} / \mathrm{s}$, respectively. The tracking accuracy of the proposed control system is enhanced and almost frees from the disturbance of the leading vehicle's acceleration/deceleration. However, for the hierarchical control system, it is affected obviously by the change of the leading vehicle's acceleration/ deceleration, and touches the maximum value of $\pm 0.1 \mathrm{~m}$. Finally, the comparison between (e) and (f) in Figures 11 and 12 demonstrates a superior robustness for the proposed control system in spite of the uncertainties caused by the road grade, gear position and the vehicle mass. Particularly, while the road grade changes between $\pm 3^{\circ}$ in the time period of $80 \mathrm{~s} \sim 120 \mathrm{~s}$, the tracking error of the relative distance and the relative velocity for the proposed control system are less than $\pm 0.05 \mathrm{~m}$ and $-0.04 \mathrm{~m} / \mathrm{s} \sim 0.02 \mathrm{~m} / \mathrm{s}$, in contract to larger than $\pm 0.15 \mathrm{~m}$ and $\pm 0.05 \mathrm{~m} / \mathrm{s}$ of the hierarchical control system.

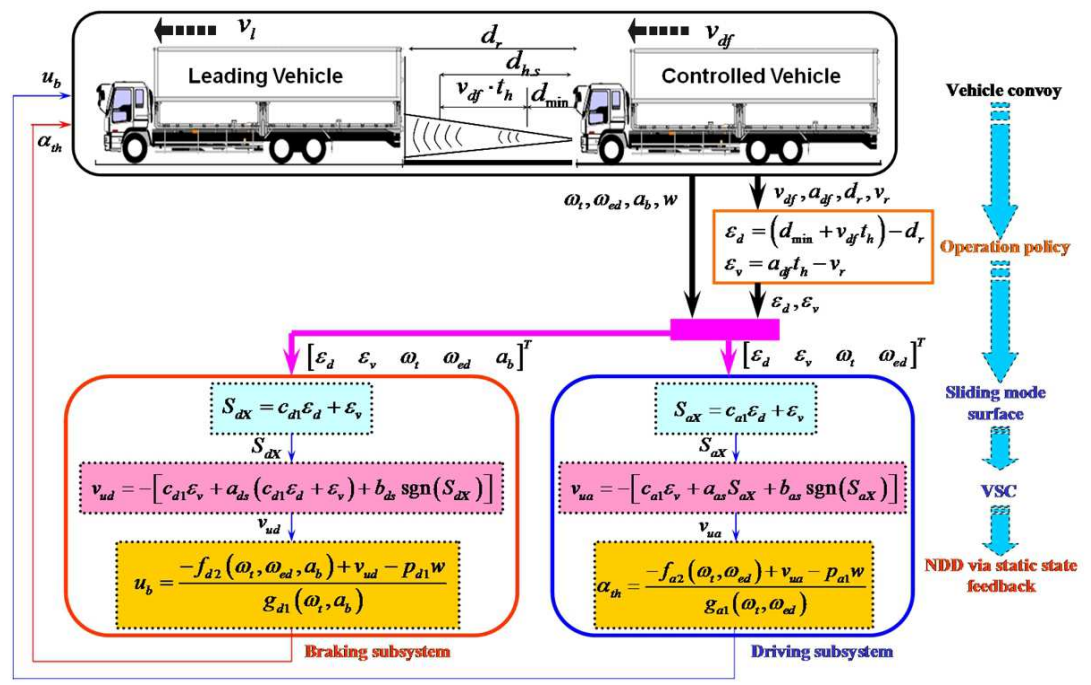

Fig. 9. LFS ACC system using the DDRC method
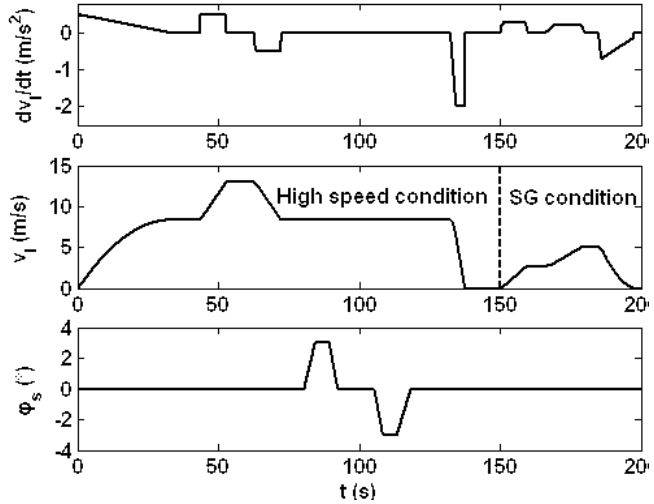

Fig. 10. Profile of leading vehicle driving cycle and road grade 
(a)

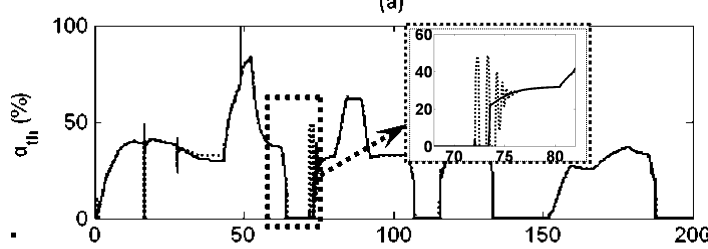

(b)

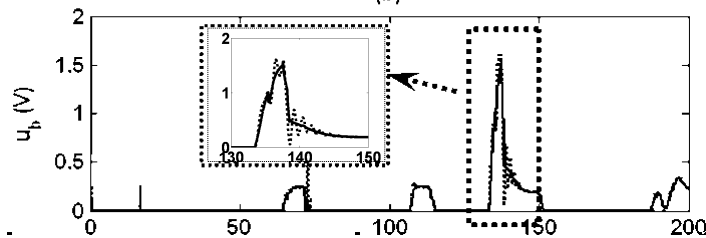

(c)

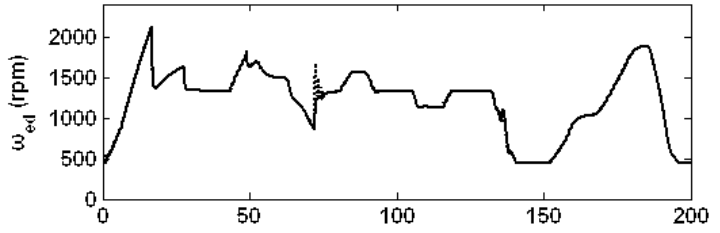

(d)

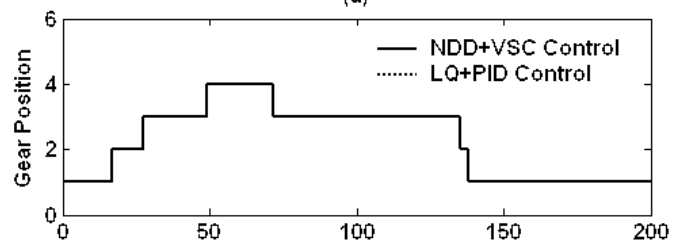

(e)
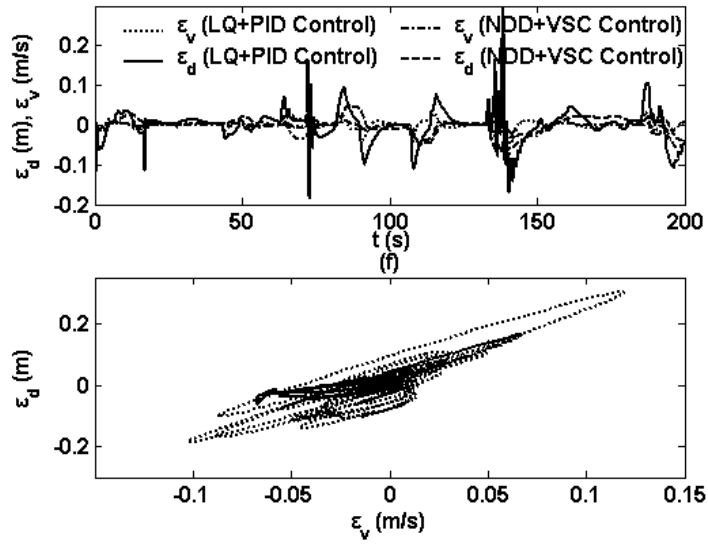

Fig. 11. Simulation results (mass is $10,000 \mathrm{~kg}$ ) 
(a)

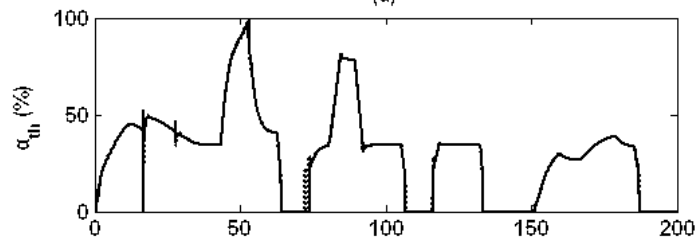

(b)

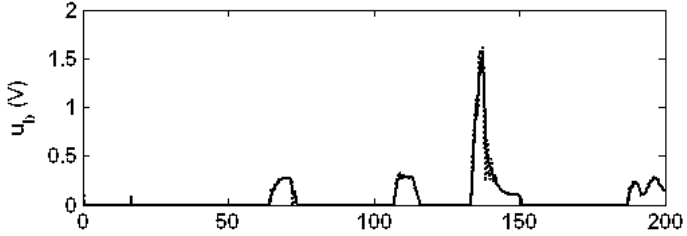

(c)

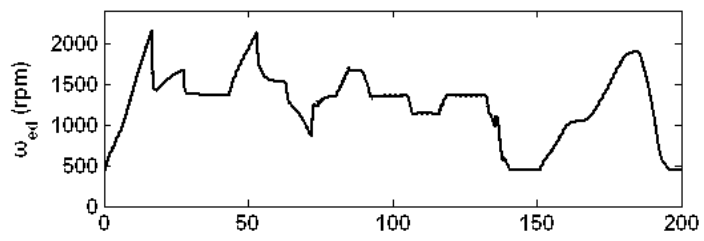

(d)

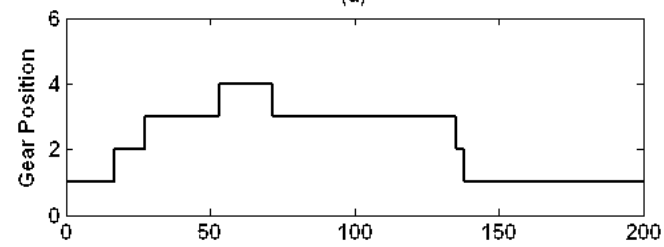

(e)
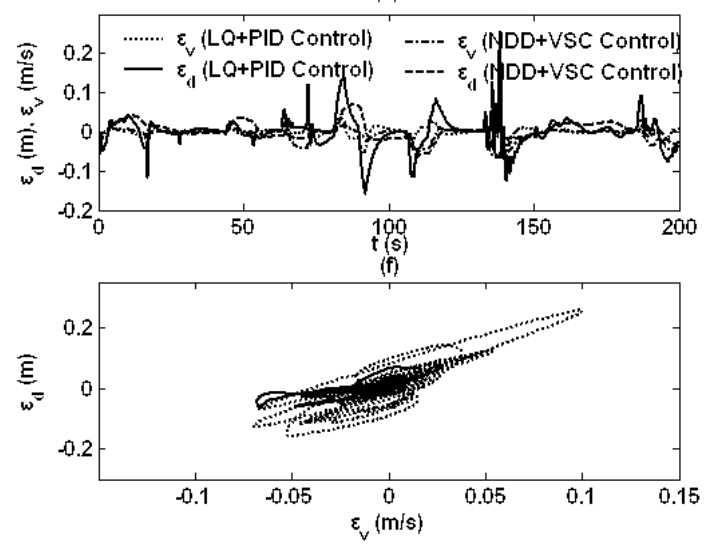

Fig. 12. Simulation results (mass is $25,000 \mathrm{~kg}$ ) 
From above analysis and the simulation results, it seems that the influence of nonlinearity, external disturbance and the variable uncertainties have been eliminated by adopting the proposed DDRC method for the LFS AACC system, and it results in a significant improvement of the tracking accuracy, robustness, as well as the response characteristics of the actuator system (i.e., the throttle angle and the EBS desired input voltage). In addition, the control structure and the parameters are simplified, and easy to determine in comparison with the hierarchical control algorithm.

\section{Conclusion}

In this study, an LFS nonlinear dynamics model is proposed by integrating the dynamics of the inter-vehicles and the controlled vehicle. Then, a DDRC method is developed, and used to design the LFS AACC system. Finally, the control performance is verified by the numerical simulation under a typical driving cycle. The simulation results confirm the followings:

1. The proposed LFS model not only can describe the vehicle's strong nonlinearity at lowspeed conditions and the uncertainty induced by the complex traffic environment and the road condition, but also is able to express the strong coupling characteristics due to frequent change of the leading vehicle's acceleration/deceleration at high-speed condition. Particularly, the dynamics of the inter-vehicles and the controlled vehicle are lumped together within a universal state space equation.

2. The tracking accuracies at high-speed and low-speed SG condition, as well as the robustness to the external disturbance and the model parameter uncertainty have been improved simultaneously, because the DDRC method is applied in the design of the LFS ACC system.

3. The actuators' high frequency oscillation caused by the unmodeled part has been restrained through using small parameters, and this leads to a control system with simplified structure.

\section{Appendixes}

Appendix 1. Definition of the matrix items in Eq. (1)

$$
\begin{aligned}
& f_{a v 1}(X)=\left\{\left[\tau_{1}\left(\frac{\omega_{t}}{\omega_{e d}}\right)^{2}+\tau_{2}\left(\frac{\omega_{t}}{\omega_{e d}}\right)+\tau_{3}\right]\left(\frac{\omega_{e d}}{60}\right)^{2} \frac{t_{1}\left(\frac{\omega_{t}}{\omega_{e d}}\right)+t_{2}}{g_{g}} \frac{i_{g_{1}} i_{0} \eta_{k}}{r_{t}}-\frac{\eta_{1}\left(\frac{\omega_{t}}{\eta_{2}}\right)^{2}+M\left(\eta_{r}+\sin \varphi_{s}\right) g_{g}}{g_{g}}\right\} \frac{i_{g_{1}} i_{0}}{\sigma_{C}} \\
& f_{a v 2}(X)=\left\{\left(k_{2} \omega_{e d}+k_{4}\right) g_{g}-\left[\tau_{1}\left(\frac{\omega_{t}}{\omega_{e d}}\right)^{2}+\tau_{2}\left(\frac{\omega_{t}}{\omega_{e d}}\right)+\tau_{3}\right]\left(\frac{\omega_{e d}}{60}\right)^{2}\right\} \frac{\eta_{2}}{I_{e}} \\
& g_{a v 1}(X)=0 \\
& g_{a v 2}(X)=\left(k_{1} \omega_{e d}+k_{3}\right) \frac{g_{g} \eta_{2}}{I_{e}}
\end{aligned}
$$


Appendix 2. Definition of the matrix items in Eq. (2)

$$
\begin{aligned}
& f_{d v 1}(X)=\left\{\left[\tau_{d 1}\left(\frac{\omega_{t}}{\omega_{e d}}\right)^{2}+\tau_{d 2}\left(\frac{\omega_{t}}{\omega_{e d}}\right)+\tau_{d 3}\right]\left(\frac{\omega_{e d}}{60}\right)^{2} \frac{i_{g_{1}} i_{0} \eta_{k}}{r_{t}}+M a_{b}-\frac{\eta_{1}\left(\frac{\omega_{t}}{\eta_{2}}\right)^{2}+M\left(\eta_{r}+\sin \varphi_{s}\right) g_{g}}{g_{g}}\right\} \frac{i_{g_{1}} i_{0}}{\sigma_{C}} \\
& f_{d v 2}(X)=\left\{\left(k_{2} \omega_{e d}+k_{4}\right) g_{g}-\left[\tau_{d 1}\left(\frac{\omega_{t}}{\omega_{e d}}\right)^{2}+\tau_{d 2}\left(\frac{\omega_{t}}{\omega_{e d}}\right)+\tau_{d 3}\right]\left(\frac{\omega_{e d}}{60}\right)^{2}\right\} \frac{\eta_{2}}{I_{e}} \\
& f_{d v 3}(X)=-\frac{1}{t_{r}} a_{b} \\
& g_{d v 1}(X)=g_{d v 2}(X)=0 \\
& g_{d v 3}(X)=-\frac{k_{b}+k_{a} a_{b}^{2} v}{t_{r}}
\end{aligned}
$$

Appendix 3. Definition of the matrix items in Eq. (7)

$$
\begin{aligned}
& f_{a 1}\left(\varepsilon_{v}\right)=\varepsilon_{v} \\
& f_{a 2}\left(\omega_{t}, \omega_{e d}\right)=\left[\alpha_{n} t_{h}\left(2 a \omega_{t}+b \omega_{e d}+3 d \frac{\omega_{t}^{2}}{\omega_{e d}}\right)+\alpha_{n}\right] \dot{\omega}_{t}+\alpha_{n} t_{h}\left(b \omega_{t}+2 c \omega_{e d}-d \frac{\omega_{t}^{3}}{\omega_{e d}^{2}}\right) \\
& \left(e \omega_{t}^{2}+f \omega_{t} \omega_{e d}+g \omega_{e d}^{2}+h \omega_{e d}+i\right) \\
& f_{a 3}\left(\omega_{t}, \omega_{e d}\right)=a \omega_{t}^{2}+b \omega_{t} \omega_{e d}+c \omega_{e d}^{2}+d \frac{\omega_{t}^{3}}{\omega_{e d}}-\vartheta \\
& f_{a 4}\left(\omega_{t}, \omega_{e d}\right)=e \omega_{t}^{2}+f \omega_{t} \omega_{e d}+g \omega_{e d}^{2}+h \omega_{e d}+i \\
& g_{a 1}\left(\omega_{t}, \omega_{e d}\right)=\alpha_{n} t_{h}\left(b \omega_{t}+2 c \omega_{e d}-d \frac{\omega_{t}^{3}}{\omega_{e d}^{2}}\right)\left(j \omega_{e d}+k\right) \\
& g_{a 2}\left(\omega_{e d}\right)=\left(j \omega_{e d}+k\right) \\
& p_{a 1}=-1 \\
& \Delta f_{a 2}\left(\omega_{t}, \omega_{e d}\right)=\left[\alpha_{n} t_{h}\left(2 a \omega_{t}+b \omega_{e d}+3 d \frac{\omega_{t}^{2}}{\omega_{e d}}\right)+\alpha_{n}\right] \Delta f_{a v 1}+\alpha_{n} t_{h}\left(b \omega_{t}+2 c \omega_{e d}-d \frac{\omega_{t}^{3}}{\omega_{e d}^{2}}\right) \Delta f_{a v 2} \\
& \Delta g_{a 1}\left(\omega_{t}, \omega_{e d}\right)=\alpha_{n} t_{h}\left(b \omega_{t}+2 c \omega_{e d}-d \frac{\omega_{t}^{3}}{\omega_{e d}^{2}}\right) \Delta g_{a v 2} \\
& \Delta f_{a 3}=\Delta f_{a v 1}, \Delta f_{a 4}=\Delta f_{a v 2}, \Delta g_{a 2}=\Delta g_{a v 2}
\end{aligned}
$$

where $a, b, c, d, e, f, g, h, i, j, k, \vartheta$ are constant coefficients, their specific values can be referred to ref. [16]. 
Appendix 4. Definition of the matrix items in Eq. (8)

$$
\begin{aligned}
& f_{d 1}\left(\varepsilon_{v}\right)=\varepsilon_{v} \\
& f_{d 2}\left(\omega_{t}, \omega_{e d}, a_{b}\right)=\left[\alpha_{n} t_{h}\left(2 a_{d} \omega_{t}+b_{d} \omega_{e d}\right)+\alpha_{n}\right] \dot{\omega}_{t}+\alpha_{n} t_{h}\left(b_{d} \omega_{t}+2 c_{d} \omega_{e d}\right) \dot{\omega}_{e d}+\alpha_{n} t_{h} d_{d} j_{d} a_{b} \\
& f_{d 3}\left(\omega_{t}, \omega_{e d}, a_{b}\right)=a_{d} \omega_{t}^{2}+b_{d} \omega_{t} \omega_{e d}+c_{d} \omega_{e d}^{2}+d_{d} a_{b}-\vartheta_{d} \\
& f_{d 4}\left(\omega_{t}, \omega_{e d}\right)=e_{d} \omega_{t}^{2}+f_{d} \omega_{t} \omega_{e d}+g_{d} \omega_{e d}^{2}+h_{d} \omega_{e d}+i_{d} \\
& f_{d 5}\left(a_{b}\right)=j_{d} a_{b} \\
& g_{d 1}\left(\omega_{t}, a_{b}\right)=\alpha_{n} t_{h} d_{d}\left(k_{d} a_{b}^{2} \omega_{t}+l_{d}\right) \\
& g_{d 2}\left(\omega_{t}, a_{b}\right)=k_{d} a_{b}^{2} \omega_{t}+l_{d} \\
& p_{d 1}=-1 \\
& \Delta f_{d 2}\left(\omega_{t}, \omega_{e d}\right)=\left[\alpha_{n} t_{h}\left(2 a_{d} \omega_{t}+b_{d} \omega_{e d}\right)+\alpha_{n}\right] \Delta f_{d v 1}+\alpha_{n} t_{h}\left(b_{d} \omega_{t}+2 c_{d} \omega_{e d}\right) \Delta f_{d v 2}+\alpha_{n} t_{h} d_{d} \Delta f_{d v 3} \\
& \Delta g_{d 1}\left(\omega_{t}, a_{b}\right)=\alpha_{n} t_{h} d_{d}\left(k_{d} a_{b}^{2} \omega_{t}+l_{d}\right) \Delta g_{d v 3} \\
& \Delta f_{d 3}=\Delta f_{d v 1}, \Delta f_{d 4}=\Delta f_{d v 2}, \Delta f_{d 5}=\Delta f_{d v 3}, \Delta g_{d 2}=\Delta g_{d v 3}
\end{aligned}
$$

where $a_{d}, b_{d}, c_{d}, d_{d}, e_{d}, f_{d}, g_{d}, h_{d}, i_{d}, j_{d}, k_{d}, l_{d}, \vartheta_{d}$ are constant coefficients, their specific values can be referred to ref. [16].

Appendix 5. The vehicle parameters are as follows:

$i_{0}=5.571$ - final reduction ratio;

$i_{g_{1}}=3.49$ - first position gear ratio of the automatic transmission;

$r_{t}=0.507$ - effective tire radius $(\mathrm{m})$;

$I_{e}=3.189$ - rotational inertia of the engine flywheel $\left(\mathrm{kg} \mathrm{m}^{2}\right) ;$

$\eta_{k}=0.98$ - total transmission efficiency;

$\eta_{r}=0.01$ - rolling resistance coefficient;

$M=10,000$ - vehicle nominal mass $(\mathrm{kg})$;

$\varphi_{s}=0$ - nominal road grade $\left({ }^{\circ}\right)$;

$k_{1}=5.2 \times 10^{-3}, k_{2}=-0.25, k_{3}=-1.1, k_{4}=145$ - engine fitting coefficients;

$t_{1}=-0.85, t_{2}=1.75, \tau_{1}=-7.19 \times 10^{-2}, \tau_{2}=3.97 \times 10^{-2} \tau_{3}=3.68 \times 10^{-2}$ - torque converter fitting coefficients for the forward transmit condition;

$\tau_{d 1}=-2.1 \times 10^{-2}, \tau_{d 2}=6.76 \times 10^{-2}, \tau_{d 3}=-4.59 \times 10^{-2}$ - torque converter fitting coefficients for the reverse transmit condition;

$t_{r}=0.2$ - time constant of the dynamic response for the braking system;

$k_{a}=-4.3 \times 10^{-3}, k_{b}=0.29$ - fitting coefficients of the heat fading efficiency model for the braking system;

$\eta_{1}, \eta_{2}, \sigma_{C}$ - constant coefficients:

$\eta_{1}=\left(0.232 g_{g}\right)\left(\frac{0.377 r \cdot 60}{3.6 i_{g} i_{0} \cdot 2 \pi}\right)^{2}, \eta_{2}=\frac{60}{6.283}, \sigma_{C}=\left(\frac{M}{g_{g} \cdot 18.8}\right)+\left(\frac{M \cdot 1.55}{g_{g}^{2} \cdot 18.8}\right) ;$

$g_{g}=9.8 \mathrm{~m} / \mathrm{s}^{2}$. 


\section{Acknowledgment}

We would like to thanks the data of vehicle supported by Tsinghua University, and the supports from the Young Scientists Fund of National Natural Science Foundation of China (51007003) and National Natural Science Foundation of China (51075010).

\section{References}

[1] Hedrick, J.K.; McMahon, D.H.; Swaroop, D. (1993). Vehicle Modeling and Control for Automated Highway Systems, PATH Technical Report, pp.1-73,UBC-ITS-PRR-93-24, Jan 01,1993

[2] Hedrick, J.K. (1998). Nonlinear Controller Design for Automated Vehicle Application, Control '98. UKACC International Conference on (Conf. Publ. No. 455), pp.2332,Swansea, UK, Sep 1-4,1998

[3] Rajamani, R.; Tan, H. S.; Law, B. K. and Zhang, W. B. (2000). Demonstration of Integrated Longitudinal and Lateral Control for the Operation of Automated Vehicles in Platoons. IEEE Transactions on Control Systems Technology, Vol.8, No.4, (July 2000), pp. 695-708, ISSN 1063-6536

[4] Yi, K.; Moon, I.; Kwon, Y. D. (2001). A vehicle-to-vehicle distance control algorithm for stop-and-go cruise control. Intelligent Transportation Systems, 2001. Proceedings. 2001 IEEE, pp. 478-482, ISBN: 0-7803-7194-1, Oakland, CA, USA, 2001

[5] Omae, M. (1999). Study on the vehicle platoon control system, Doctor Dissertation, Tokyo: University of Tokyo, 1999

[6] Fritz, A.; Schienlen, W. (1999). Automatic Cruise Control of a Mechatronically Steered Vehicle Convoy. Vehicle System Dynamics, Vol.32, (1999), pp. 331-344

[7] Schienlen, W.; Fritz, A. (1999). Nonlinear Cruise Control Concepts for Vehicle in Convoy. Vehicle System Dynamics Supplement, Vol.33,(1999), pp.256-269

[8] Naranjo, J.E.; Gonzalez, C.; Garcia, R.; de Pedro, T. (2006). ACC+Stop\&go maneuvers with throttle and brake fuzzy control. IEEE Transactions on Intelligent Transportation Systems, Vol.7 ,No.4,(2006), pp. 213-225, ISSN: 1524-9050

[9] Daniele, C.; Schutter, B. De. (2008). Adaptive Cruise Control for a SMART Car: A Comparison Benchmark for MPC-PWA Control Methods. IEEE Transactions on Control Systems Technology, Vol.16, No.2, (March 2008), pp. 365-372, ISSN: 1063-6536

[10] Bifulco, G.N.; Simonelli, F.; Di Pace, R. (2008). Experiments toward a human-like adaptive cruise control, Intelligent Vehicles Symposium, pp. 919-924, ISBN 978-14244-2568-6, Eindhoven University of Technology, Eindhoven, The Netherlands, June 4-6, 2008

[11] Acarman, T.; Liu, Y. and Ozguner, U. (2006). Intelligent cruise control stop and go with and without communication, Proceedings of the American Control Conference, pp. 4356-4361, ISBN: 1-4244-0209-3, Minneapolis, Minnesota, USA, June 14-16, 2006

[12] Martinez, J. J. and Canudas-de-Wit, C. (2007). A Safe Longitudinal Control for Adaptive Cruise Control and Stop-and-Go Scenarios. IEEE Transactions on Control Systems Technology, Vol.15, No.2, (March 2007), pp.246-258, ISSN : 1063-6536

[13] Venhovens, P.; Naab, K.; Adiprasito, B. (2000). Stop and go cruise control, Proceedings of Seoul 2000 FISITA World Automotive Congress, pp.1-8, Seoul Korea, June 12-15, 2000 
[14] Yamamura, Y.; Tabe, M.; Kanehira, M. (2001). Development of an adaptive cruise control system with stop and go capability,SAE Technical Paper, Detroit, MI, USA, March, 2001

[15] Bin,Y; Li, K. Q.; Ukawa, H.; Handa, M. (2006). Modeling and Control of Nonlinear Dynamic System for Heavy-Duty Trucks. Proceedings of the Institution of Mechanical Engineers, Part D, Journal of Automobile Engineering, Vol.220, No.10, Z(2006), pp. 1423-1435

[16] Bin, Y.; Li, K. Q.; Feng, N. L. (2008). Feedback Linearization Tracking Control of Vehicle Longitudinal Acceleration under Low-Speed Conditions. Journal of Dynamic Systems, Measurement, and Control, Vol.130, (2008), pp. 1-12

[17] Li, K. Q.; Bin, Y.; Ukawa, H.; Handa ,M. (2006). Study on Stop and Go Cruise Control of Heavy-Duty Vehicles. Transaction of JSAE, Vol.37, No.2, (2006), pp. 145-150, ISSN:0919-1364

[18] Bin,Y.; Li, K. Q; Ukawa, H.; Handa, M. (2009). Nonlinear Disturbance Decoupling Control of Heavy-Duty Truck Stop and Go Cruise Systems. Vehicle System Dynamics, Vol.47, No.1, (January 2009), pp. 29-55

[19] Thomas D. Gillespie. (1992). Fundamentals of Vehicle Dynamics, Warrendale, Society of Automotive Engineers, Inc, 1992

[20] Swaroop, D.; Hetirick, J.K.; Chien, C.C.; Ioannou, P. (2004). A Comparison of Spacing and Headway Control Laws for Automatically Controlled Vehicles. Vehicle System Dynamics, Vol.23, No.8, (2004), pp. 597-625

[21] Bengtsson, J. (2001). Adaptive Cruise Control and Driver Modeling. Research thesis, Department of automatic control, Lund Institute of Technology, ISSN 0280-5316, Sweden

[22] Isidori, A.; Krener, A.; Gori-Giorgi, C.; Monaco, S. (1981). Nonlinear Decoupling via Feedback: A Differential Geometric Approach. IEEE Transactions on Automatic Control, Vol.26, No.2, (1981), pp. 331-345, ISSN: 0018-9286

[23] Nijmeijer, H. (1994). On dynamic state feedback in nonlinear control, IEE Colloquium on Nonlinear Control, pp.5/1 5/2, London, UK, May 24,1994

[24] Xia, X H. (1997). Disturbance Decoupling Control. Science Press, Beijing, 1997

[25] Morse, A.; Wonham, W. (1971). Status of noninteracting control. IEEE Transactions on Automatic Control, Vol.16, No.6, (Dec1971), pp. 568-581, ISSN: 0018-9286

[26] Conte, G.; Perdon, A. M. (1994). The disturbance decoupling problem by dynamic feedback for systems over a principal ideal domain, Proceedings of the 33rd IEEE Conference on Decision and Control, pp. 1276-1279, ISBN: 0-7803-1968-0, Lake Buena Vista, FL , USA, Dec 14-16,1994

[27] Xia, X H. (1993). Companion of Controlled invariant Distribution and Description of DDP Control Law, Science in China, pp. 130-136, Ser.A, 1993,

[28] Young, K.D. (1999). Variable structure systems, sliding mode and nonlinear control, Springer, London, 1999

[29] Hu, Y. M. (2003). Variable Structure Control Theory and Application, Science Press, Beijing

[30] Cheng, D. Z. (1988). Geometric Theory of Nonlinear System. Science Press, Beijing

[31] Byrnes, C.I.; Isidori, A.(1998). Output regulation for nonlinear systems: an overview, Proceedings of the 37th IEEE Conference on Decision and Control, pp.3069-3074, Tampa, FL, UNITED STATES, Dec 16-18, 1998

[32] Fliess, M.; Tevine, J.; Martin, P.; Rouchon, P.(1994). Nonlinear control and LieBacklund transformations: towards a new differential geometric standpoint, 1994 
Proceedings of the 33rd IEEE Conference on Decision and Control, pp.339-344, ISBN 07803-1968-0, Lake Buena Vista, FL , USA , Dec 14-16,1994

[33] Cheng, D. Z. (1987). Geometric Approach to Nonlinear Systems Part 1. Geometric Method and Geometric Preliminary, Control Theory and Application, Jan 1-9,1987

[34] Han, Z. Z.; Liu, J. H, et al.(1994). Characters of Nonlinear Control Systems (II). Control and Decision, Vol.9, No.5, (1994), pp. 91-97

[35] Wang, Y. F.; Xia, X. H.; Gao, W. B. (1994). Parameter Variations in Nonlinear Decoupling, American Control Conference, pp. 2700 - 2704 , ISBN: 0-7803-1783-1, June 29- July 1, 1994

[36] Isidori, A. (1985). Nonlinear Control Systems, Spronger Verlag,ISBN 0-387-15595-3, Berlin, Heidelberg

[37] Gao, W. B. (1988). Nonlinear Control System Introduction. Science Press, Beijing

[38] Slotine, J.; Li, W. (1991). Applied Nonlinear Control, Prentice-Hall Inc, ISBN 0-13-0408905,Englewood Cliffs, New Jersey 


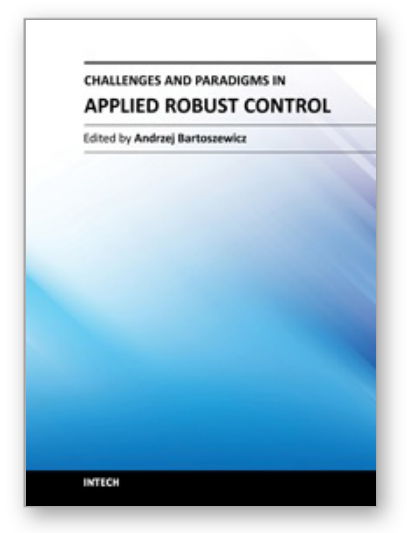

\author{
Challenges and Paradigms in Applied Robust Control \\ Edited by Prof. Andrzej Bartoszewicz
}

ISBN 978-953-307-338-5

Hard cover, 460 pages

Publisher InTech

Published online 16, November, 2011

Published in print edition November, 2011

The main objective of this book is to present important challenges and paradigms in the field of applied robust control design and implementation. Book contains a broad range of well worked out, recent application studies which include but are not limited to $\mathrm{H}$-infinity, sliding mode, robust PID and fault tolerant based control systems. The contributions enrich the current state of the art, and encourage new applications of robust control techniques in various engineering and non-engineering systems.

\title{
How to reference
}

In order to correctly reference this scholarly work, feel free to copy and paste the following:

Yang Bin, Keqiang Li and Nenglian Feng (2011). Modelling and Nonlinear Robust Control of Longitudinal Vehicle Advanced ACC Systems, Challenges and Paradigms in Applied Robust Control, Prof. Andrzej Bartoszewicz (Ed.), ISBN: 978-953-307-338-5, InTech, Available from:

http://www.intechopen.com/books/challenges-and-paradigms-in-applied-robust-control/modelling-andnonlinear-robust-control-of-longitudinal-vehicle-advanced-acc-systems

\section{INTECH}

open science | open minds

\section{InTech Europe}

University Campus STeP Ri

Slavka Krautzeka 83/A

51000 Rijeka, Croatia

Phone: +385 (51) 770447

Fax: +385 (51) 686166

www.intechopen.com

\section{InTech China}

Unit 405, Office Block, Hotel Equatorial Shanghai

No.65, Yan An Road (West), Shanghai, 200040, China

中国上海市延安西路65号上海国际贵都大饭店办公楼 405 单元

Phone: +86-21-62489820

Fax: +86-21-62489821 
(C) 2011 The Author(s). Licensee IntechOpen. This is an open access article distributed under the terms of the Creative Commons Attribution 3.0 License, which permits unrestricted use, distribution, and reproduction in any medium, provided the original work is properly cited. 